\title{
Lifting of Quaternionic Frames to Higher Dimensions with Partial Ridges
}

\author{
Florian Heinrich*® and Brigitte Forster
}

\begin{abstract}
We consider the technique of lifting frames to higher dimensions with the ridge idea that originally was introduced by Grafakos and Sansing. We pursue a novel approach with regard to a non-commutative setting, concretely the skew-field of quaternions. Moreover, we allow for splitting dimensions and for lifting with regard to multi-ridges. To this end, we introduce quaternionic Sobolev spaces and prove the corresponding embedding theorems. We mention as concrete examples quaternionic wavelet frames and quaternionic shearlet frames, and give the respective lifted families.
\end{abstract}

Mathematics Subject Classification. Primary 42C15, Secondary 42C40.

Keywords. Quaterionic frames, (Quaternionic) Frame lifting, Quaternionic Sobolev spaces, Quaternionic Multivariate Radon transform, Fourier multi-slice theorem, Ridge functions.

\section{Introduction}

Calculating frame bounds can be tedious. A beautiful solution to lift Gabor frames to higher dimension while keeping the frame bounds (up to a factor 2) was introduced by Grafakos and Sansing [17]. They applied ridge functions to one-dimensional frames to lift them to higher dimensional semi-discrete function families with properties similar to frames. The idea can also be extended to other frames than Gabor frames, e.g. wavelet frames [10]. In this article, we extend this technique of lifting frames to higher dimensions to the case of the function spaces based on the non-commutative division ring of quaternionic numbers $\mathbb{H}$. We also consider partial ridges and give a precise description on their influence on the frame bounds for these methods of frame extension to higher dimensions.

This article is part of the Topical Collection on ISAAC 12 at Aveiro, July 29-August 2, 2019, edited by Swanhild Bernstein, Uwe Kaehler, Irene Sabadini, and Franciscus Sommen.

*Corresponding author. 
There have been several constructions of quaternionic or hypercomplex frames for image processing, e.g. quaternionic Gabor filters [4], wavelet frames (for an overview see [16]) and in particular steerable wavelet frames [21,34]. In the image processing literature the quaternionic Fourier transforms are mainly generated by raising the complex exponential kernel to a quaternionic power. This results in a variety of possible Fourier transforms, see e.g., $[6,7,13,16,30]$ for an overview in the wavelet case. However, these Fourier transform operators differ from the Fourier transform in Clifford Analysis as it has been defined by Brackx, De Schepper, and Sommen [12]. Their so-called Clifford-Fourier transform diagonalizes the Laplace operator and therefore perfectly fits the aims of Harmonic Analysis. Sobolev spaces and also wavelets have been introduced for the Clifford-Fourier transform [5, 23]. However note, they do not rely on the same definition of the Fourier transform as it is widely use in image processing. We here have two different areas of quaternionic analysis. A beautiful description of these two research streams can be found in the book [22, p. xi-xxvii]. In this article, we add to the first area of quaternionic analysis as we aim at the applicability to image processing. We show the similarities and the differences of the various Fourier transforms. To the best of our knowledge, there has been no description of the corresponding Sobolev spaces in the literature so far. In our article we define the Sobolev spaces via the Fourier transforms as this allows for a nondiscrete analysis of the regularity and for fractional differential operators. The resulting spaces often are also called Bessel potential spaces [32].

We briefly revisit the skew field of quaternions $\mathbb{H}$ : Let $i, j, k$ be imaginary units satisfying the properties $i^{2}=j^{2}=k^{2}=i j k=-1$. An element $q=$ $a+b i+c j+d k \in \mathbb{H}$ can be split into a scalar part $\mathrm{S}(q):=a$ and a vector part $\mathrm{V}(q):=b i+c j+d k$. If $q$ is of the form $q=a+0 i+0 j+0 k$, then it is called real. If $a=0$ and at least one of $b, c, d \neq 0$, then $q$ is called purely imaginary. The additive group of pure quaternions together with the zero element is denoted by $\mathrm{V}(\mathbb{H})$. The conjugation of complex numbers can be generalized using the scalar and the vector part $\bar{q}=S(q)-V(q)$. But it has slightly different properties than its complex analog. The most remarkable difference is its antiautomorphism property $\overline{p q}=\bar{q} \cdot \bar{p}$.

The modulus $|q|:=\sqrt{a^{2}+b^{2}+c^{2}+d^{2}}$ is defined as on $\mathbb{R}^{4}$ and is compatible with multiplication, i.e., $|p q|=|p||q|$ for two quaternions $p, q \in \mathbb{H}$. If $|q|=1$, then $q$ is called a unit quaternion. For further details on quaternions we refer to [11] and [15, Chapter 1].

For our endeavor, we consider the following function spaces over $\mathbb{R}^{n}$, $n \in \mathbb{N}: \mathcal{C}^{\ell}\left(\mathbb{R}^{n} ; \mathbb{C}\right), \ell \in \mathbb{N}$, denotes the vector space of all complex valued $\ell$-times continuously differentiable functions. The space of infinitely times differentiable functions is defined as

$$
\mathcal{C}^{\infty}\left(\mathbb{R}^{n} ; \mathbb{C}\right):=\bigcap_{\ell=0}^{\infty} \mathcal{C}^{\ell}\left(\mathbb{R}^{n} ; \mathbb{C}\right) .
$$

For $1 \leq p<\infty, L^{p}\left(\mathbb{R}^{n} ; \mathbb{H}\right)$ denotes the vector space of $p$-integrable, quaternion valued functions. Their norms are denoted by $\|\cdot\|_{p}$. For $p=2$, 
the norm induces an inner product

$$
\langle\cdot, \cdot\rangle: L^{2}\left(\mathbb{R}^{n} ; \mathbb{H}\right) \times L^{2}\left(\mathbb{R}^{n} ; \mathbb{H}\right) \rightarrow \mathbb{H},\langle f, g\rangle:=\int_{\mathbb{R}^{n}} f(x) \cdot \overline{g(x)} d x .
$$

$L^{\infty}\left(\mathbb{R}^{n} ; \mathbb{H}\right)$ is the respective vector space of functions bounded almost everywhere.

The aim of our article is to extend one-dimensional quaternionic-valued frames to higher dimensions. For this, we will consider continuous frames:

Definition 1.1. (Continuous frame) Let $M$ be a measure space with a positive measure $\mu$. A family of functions $\left\{f_{\kappa}\right\}_{\kappa \in M} \subset L^{2}\left(\mathbb{R}^{n} ; \mathbb{H}\right)$ is called frame if $\kappa \mapsto\left\langle f, f_{\kappa}\right\rangle$ is measurable on $M$ and there exist constants $A, B>0$ such that

$$
A\|f\|_{2}^{2} \leq \int_{M}\left|\left\langle f, f_{\kappa}\right\rangle\right|^{2} d \mu(\kappa) \leq B\|f\|_{2}^{2}
$$

for all $f \in L^{2}\left(\mathbb{R}^{n} ; \mathbb{H}\right)$. For every continuous frame $\left\{f_{\kappa}\right\}_{\kappa \in M}$, the dual continuous frame $\left\{g_{\kappa}\right\}_{\kappa \in M}$ is defined via the following property: Every $f \in L^{2}\left(\mathbb{R}^{n}, \mathbb{H}\right)$ has the representation

$$
f=\int_{M}\left\langle f, f_{k}\right\rangle g_{k} d \mu(k)
$$

here the integral is to be interpreted in the weak sense.

For every continuous frame $\left\{f_{\kappa}\right\}_{\kappa \in M}$, there exists at least one dual continuous frame $\left\{g_{\kappa}\right\}_{\kappa \in M}$.

Remark 1.2. The more widespread "discrete" frames are defined as sequence of vectors $\left(f_{\kappa}\right)_{\kappa \in M} \subset L^{2}\left(\mathbb{R}^{n} ; \mathbb{H}\right)$ such that $M$ is countable and there exist $0<A \leq B<\infty$ such that

$$
A\|f\|_{2}^{2} \leq \sum_{\kappa \in M}\left|\left\langle f, f_{\kappa}\right\rangle\right|^{2} \leq B\|f\|_{2}^{2}
$$

for all $f \in L^{2}\left(\mathbb{R}^{n} ; \mathbb{H}\right)$ They are a special case of Definition 1.1. This means that the whole classical frame theory and all its special frame classes like (discrete) wavelets, shearlets and Gabor frames are included in Definition 1.1.

For more information on frames, dual frames and continuous frames, we refer to $[1,9,24]$.

For our endeavor we will apply the Fourier transform and its quaternionic extenions. The Fourier transform on $L^{1}\left(\mathbb{R}^{n} ; \mathbb{C}\right)$ is denoted by

$$
\mathcal{F}: L^{1}\left(\mathbb{R}^{n} ; \mathbb{C}\right) \rightarrow \mathcal{C}_{0}\left(\mathbb{R}^{n} ; \mathbb{C}\right), \mathcal{F} f(\omega):=\int_{\mathbb{R}^{n}} f(x) e^{-2 \pi i \omega^{T} x} d x .
$$

For $L^{2}\left(\mathbb{R}^{n} ; \mathbb{C}\right)$ the operator is extended in the usual way to $\mathcal{F}: L^{2}\left(\mathbb{R}^{n} ; \mathbb{C}\right) \rightarrow$ $L^{2}\left(\mathbb{R}^{n} ; \mathbb{C}\right)$. The corresponding inverse Fourier transform is denoted by $\mathcal{F}^{-1}$. For the basic properties of the Fourier transform we refer to [25]. 


\subsection{A closer look on the quaternions and their non-commutativity}

Now we investigate the quaternion's non-commutative character in more detail. These results will be used later on to define and to examine several variants of the Fourier transform and their relations.

The non-commutative multiplication of the quaternions will turn out to be one of the major issues. To control this problem, quaternions can be decomposed into $\mathbb{C}$-like components. We will use this later for the quaternionic Fourier transform, see e.g. [14], [15, p. 13].

Definition 1.3. (Symplectic decomposition) Let $q \in \mathbb{H}$, and $\mu, \mu_{2} \in \mathrm{V}(\mathbb{H})$ be non-zero pure quaternions. Moreover, suppose $\mu \perp \mu_{2}$.

Then the decomposition

$$
q=\underbrace{(a+b \mu)}_{=: q_{s}}+\underbrace{(c+d \mu)}_{=: q_{p}} \mu_{2}
$$

is called symplectic decomposition. $q_{s}$ is called the symplectic part and $q_{p}$ the perplectic part.

Lemma 1.4. (Properties of the symplectic decomposition) Let $q \in \mathbb{H}$ and $\mu, \mu_{2} \in \mathrm{V}(\mathbb{H})$ be pure unit quaternions such that $\mu \perp \mu_{2}$ (with respect to the $\mathbb{R}^{4}$ inner product). Then the following holds:

(i) $\mu$ and $\mu_{2}$ anticommute; i.e., $\mu \mu_{2}=-\mu_{2} \mu$.

(ii) $q_{s}=\frac{1}{2}(q-\mu q \mu)$.

(iii) $q_{p} \mu_{2}=\frac{1}{2}(q+\mu q \mu)$.

(iv) The set $\left\{1, \mu, \mu_{2}, \mu \mu_{2}\right\}$ forms a basis for $\mathbb{H}$. Thus, for a fixed pair $\mu, \mu_{2}$ there exists a unique symplectic decomposition for every $q \in \mathbb{H}$.

(v) $q=q_{s}+\mu_{2} \overline{q_{p}}$.

(vi) $|q|=\sqrt{\left|q_{s}\right|^{2}+\left|q_{p}\right|^{2}}$.

Proof. Direct calculation.

\subsection{Quaternionic Fourier Transforms}

Based on the symplectic representation of quaternions, we can introduce quaternionic Fourier transforms. Due to the non-commutative nature of the multiplication there exist multiple variants. Besides lifting the well known properties from the complex setting, it is natural to ask for relations between the different definitions. These questions will be answered in this section and exploited later on.

To this end, let $f \in L^{1}\left(\mathbb{R}^{n} ; \mathbb{H}\right)$. We consider three canonical classes as extensions of the Fourier transform. Fix $\mu, \mu_{2} \in \mathrm{V}(\mathbb{H})$ as pure unit quaternions. Let $\omega \in \mathbb{R}^{n}$.

The right version.

$$
\mathcal{F}_{R}(\omega):=\mathcal{F}_{R, \mu} f(\omega):=\int_{\mathbb{R}^{n}} f(x) e^{-2 \pi \mu \omega^{T} x} d x
$$

The left version.

$$
\mathcal{F}_{L} f(\omega):=\mathcal{F}_{L, \mu} f(\omega):=\int_{\mathbb{R}^{n}} e^{-2 \pi \mu \omega^{T} x} f(x) d x
$$


The sandwich version. For fixed $n_{1}, n_{2} \in \mathbb{N}$ such that $n=n_{1}+n_{2}$,

$$
\begin{gathered}
\mathcal{F}_{S} f\left(\omega_{1}, \omega_{2}\right):=\mathcal{F}_{S, \mu, \mu_{2}} f\left(\omega_{1}, \omega_{2}\right):=\int_{\mathbb{R}^{n}} e^{-2 \pi \mu \omega_{1}^{T} x} f(x, y) e^{-2 \pi \mu_{2} \omega_{2}^{T} y} d x d y \\
\text { where } \omega_{1} \in \mathbb{R}^{n_{1}} \text { and } \omega_{2} \in \mathbb{R}^{n_{2}} .
\end{gathered}
$$

Note that $\mathcal{F}_{R}$ ist left- $\mathbb{H}$-linear, $\mathcal{F}_{L}$ is right- $\mathbb{H}$-linear, whereas $\mathcal{F}_{S}$ is $\mathbb{R}$-linear only; see also [15, p. 35ff], [8], [3].

These three quaternionic Fourier transforms are related:

Lemma 1.5. (Fourier equalities) Let $f \in L^{1}\left(\mathbb{R}^{n} ; \mathbb{H}\right)$ and let $f=f_{s}+f_{p} \mu_{2} \in$ $L^{1}\left(\mathbb{R}^{n} ; \mathbb{H}\right)$ be the symplectic decomposition with respect to the pure unit quaternions $\mu$ and $\mu_{2}$. Then, for every $\omega \in \mathbb{R}^{n}$ :

(i) $\mathcal{F}_{L} f(\omega)=-\overline{\mathcal{F}_{R} \bar{f}(-\omega)}$.

(ii) $\left|\mathcal{F}_{L} f(\omega)\right|=\left|\mathcal{F}_{R} \bar{f}(-\omega)\right|$. In the case $f\left(\mathbb{R}^{n}\right) \subset \mathrm{V}(\mathbb{H})$,

$$
\left|\mathcal{F}_{L} f(\omega)\right|=\left|\mathcal{F}_{R} f(-\omega)\right| \text {. }
$$

(iii) It is

$$
\mathcal{F}_{S, \mu, \mu} f_{s}\left(\omega_{1}, \omega_{2}\right)=\mathcal{F}_{R, \mu} f_{s}\left(\omega_{1}, \omega_{2}\right)
$$

and

$$
\mathcal{F}_{S, \mu, \mu}\left(f_{p} \mu_{2}\right)\left(\omega_{1}, \omega_{2}\right)=\mathcal{F}_{R, \mu} f_{p}\left(\omega_{1},-\omega_{2}\right) \mu_{2},
$$

The properties (i)-(iii) can be extended to $L^{2}\left(\mathbb{R}^{n} ; \mathbb{H}\right)$ and then are valid in the $L^{2}$-sense.

Proof. This follows directly from the symplectic decomposition.

The next lemma combines the symplectic decomposition with the quaternionic various Fourier transforms. In fact, the compatibility of both concepts is not by chance but by design of the decomposition. For further reference consider [14, p. 42f].

Lemma 1.6. (Fourier transform and symplectic decomposition) Let $f=f_{s}+$ $f_{p} \mu_{2} \in L^{1}\left(\mathbb{R}^{n} ; \mathbb{H}\right)$ be the symplectic decomposition with respect to the pure quaterions $\mu$ and $\mu_{2}$. Suppose $\mu$ is the pure quaternion in the exponential kernel of the Fourier transform. Then, for every $\omega \in \mathbb{R}^{n}$ :

(i) $\left|\mathcal{F}_{R} f(\omega)\right|^{2}=\left|\mathcal{F}_{R} f_{s}(\omega)\right|^{2}+\left|\mathcal{F}_{R} f_{p}(-\omega)\right|^{2}$,

(ii) $\left|\mathcal{F}_{L} f(\omega)\right|^{2}=\left|\mathcal{F}_{L} f_{s}(\omega)\right|^{2}+\left|\mathcal{F}_{L} f_{p}(\omega)\right|^{2}$,

(iii) $\mathcal{F}_{R} f_{s}(\omega)=\mathcal{F}_{L} f_{s}(\omega)$ and $\mathcal{F}_{R} f_{p}(\omega)=\mathcal{F}_{L} f_{p}(\omega)$.

Proof. Direct calculation. Note that $e^{2 \pi \mu(-\omega)^{T} x}$ and $f_{s}(x)$ commute because they are both contained in $\mathbb{C}_{\mu}$; i.e., they are of the form $\mathbb{R}+\mu \mathbb{R}$.

\section{Quaternionic Sobolev Spaces}

An important class of functions spaces, the Sobolev spaces, are defined via the Fourier transform. For complex-valued functions, they are defined as follows.

Let $\alpha \geq 0$. The space

$$
H^{\alpha}\left(\mathbb{R}^{n} ; \mathbb{C}\right):=\left\{f \in L^{2}\left(\mathbb{R}^{n} ; \mathbb{C}\right):\left(1+|\cdot|^{2}\right)^{\frac{\alpha}{2}} \mathcal{F} f \in L^{2}\left(\mathbb{R}^{n} ; \mathbb{C}\right)\right\}
$$


is called Sobolev space.

Endowed with the norm

$$
\|\cdot\|: H^{\alpha}\left(\mathbb{R}^{n} ; \mathbb{C}\right) \rightarrow \mathbb{R}_{0}^{+},\|f\|:=\left(\int_{\mathbb{R}^{n}}\left(1+|\omega|^{2}\right)^{\alpha}|\mathcal{F} f(\omega)|^{2} d \omega\right)^{\frac{1}{2}}
$$

the space becomes a Banach space [19,26,33,35].

The purpose of this section is to generalize this concept to a quaternionic setting. There are several generalizations of the Fourier transform. Do these spaces with different Fourier transforms coincide? Which role does the pure unit quaternion play in the exponential kernel? As the Fourier transforms are part of the definition of the norms, a further question is whether the norms are equivalent, or even equal. We will also investigate the respective quaternionic linearity conditions and more general, the relation to direct sums of real Sobolev spaces, as well as embedding theorems.

Theorem 2.1. (Norm equality) Let $f \in H^{\alpha}\left(\mathbb{R}^{n} ; \mathbb{H}\right)$, where the space can be based on any version of the three Fourier transforms $\mathcal{F}_{L}, \mathcal{F}_{R}$ or $\mathcal{F}_{S}$. Then for the pure unit quaternions $\mu, \lambda$,

(i) $\|f\|_{L, \mu}=\|f\|_{R, \mu}$,

(ii) $\|f\|_{R, \mu}=\|f\|_{R, \lambda}$,

(iii) $\|f\|_{S, \mu, \mu}=\|f\|_{R, \mu}$,

(iv) $\|f\|_{S, \mu, \lambda}=\|f\|_{R, \mu}$.

Proof. (i) Direct calculation with application of the equalities in Lemmas 1.5 and 1.6 .

(ii) To prove this we express $\mu$ in terms of $\lambda$. To this end, we define the function $\kappa_{\beta}: \mathbb{H} \rightarrow \mathbb{H}, \kappa_{\beta}(x):=\beta^{-1} x \beta$ for some non-zero $\beta \in \mathbb{H}$. For any pure unit quaternion $\lambda$ there exists a $\beta \in \mathbb{H}$ such that $\kappa_{\beta}(\lambda)=\mu$. Now we consider $f \in L^{2}\left(\mathbb{R}^{n} ; \mathbb{H}\right)$.

$$
\begin{aligned}
\mathcal{F}_{R, \lambda}\left(f \beta^{-1}\right)(\omega) \beta & =\int_{\mathbb{R}^{n}} f(x) \beta^{-1} e^{-2 \pi \lambda \omega^{T} x} \beta d x \\
& =\int_{\mathbb{R}^{n}} f(x) \beta^{-1}\left(\cos \left(2 \pi \omega^{T} x\right)-\lambda \sin \left(-2 \pi \omega^{T} x\right)\right) \beta d x \\
& =\int_{\mathbb{R}^{n}} f(x)\left(\beta^{-1} \beta \cos \left(2 \pi \omega^{T} x\right)-\beta^{-1} \lambda \beta \sin \left(-2 \pi \omega^{T} x\right)\right) d x \\
& =\int_{\mathbb{R}^{n}} f(x)\left(\cos \left(2 \pi \omega^{T} x\right)-\mu \sin \left(-2 \pi \omega^{T} x\right)\right) d x \\
& =\int_{\mathbb{R}^{n}} f(x) e^{-2 \pi \mu \omega^{T} x} d x \\
& =\mathcal{F}_{R, \mu} f(\omega) \text { in } L^{2} \text {-sense. }
\end{aligned}
$$

When applying (i) twice and with the right linearity of $\mathcal{F}_{L}$, we deduce

$$
\begin{aligned}
\|f\|_{R, \lambda}^{2}=\|f\|_{L, \lambda}^{2} & =|\beta|^{2} \int_{\mathbb{R}^{n}}\left(1+|\omega|^{2}\right)^{\alpha}\left|\mathcal{F}_{L}\left(f \beta^{-1}\right)(\omega)\right|^{2} d \omega \\
& =|\beta|^{2}\left\|f \beta^{-1}\right\|_{L, \lambda}^{2}=|\beta|^{2}\left\|f \beta^{-1}\right\|_{R, \lambda}^{2} .
\end{aligned}
$$


Hence

$$
\begin{aligned}
\|f\|_{R, \lambda}^{2} & =|\beta|^{2}\left\|f \beta^{-1}\right\|_{R, \lambda}^{2} \\
& =|\beta|^{2} \int_{\mathbb{R}^{n}}\left(1+|\omega|^{2}\right)^{\alpha}|\beta|^{-2}\left|\mathcal{F}_{R, \lambda}\left(f \beta^{-1}\right)(\omega) \beta\right|^{2} d \omega \\
& =|\beta|^{2} \int_{\mathbb{R}^{n}}\left(1+|\omega|^{2}\right)^{\alpha}|\beta|^{-2}\left|\mathcal{F}_{R, \mu} f(\omega)\right|^{2} d \omega \\
& =\int_{\mathbb{R}^{n}}\left(1+|\omega|^{2}\right)^{\alpha}\left|\mathcal{F}_{R, \mu} f(\omega)\right|^{2} d \omega=\|f\|_{R, \mu}^{2}
\end{aligned}
$$

which proves the claim.

(iii) Direct calculation by using the symplectic decomposition with respect to $\mu, \mu_{2}$ and Lemma 1.5 .

(iv) Apply (iii) and the technique in (ii) to rotate $\lambda$ to $\mu$.

An immediate consequence is the following Corollary:

Corollary 2.2. (Set equivalence) Let $n \geq 2, \alpha \geq 0, f \in L^{2}\left(\mathbb{R}^{n} ; \mathbb{H}\right)$. Fix the pure unit quaternions $\mu, \mu_{2} \in \mathrm{V}(\mathbb{H})$. Then the following three properties are equivalent.

(i) $\left(1+|\cdot|^{2}\right)^{\frac{\alpha}{2}} \mathcal{F}_{R} f \in L^{2}\left(\mathbb{R}^{n} ; \mathbb{H}\right)$.

(ii) $\left(1+|\cdot|^{2}\right)^{\frac{\alpha}{2}} \mathcal{F}_{L} f \in L^{2}\left(\mathbb{R}^{n} ; \mathbb{H}\right)$.

(iii) $\left(1+|\cdot|^{2}\right)^{\frac{\alpha}{2}} \mathcal{F}_{S} f \in L^{2}\left(\mathbb{R}^{n} ; \mathbb{H}\right)$.

Moreover, for the left or right quaternionic Fourier transform and two pure quaternions $\mu, \lambda \in \mathrm{V}(\mathbb{H})$,

$$
\left(1+|\cdot|^{2}\right)^{\frac{\alpha}{2}} \mathcal{F}_{L / R, \mu} f \in L^{2}\left(\mathbb{R}^{n} ; \mathbb{H}\right) \Leftrightarrow\left(1+|\cdot|^{2}\right)^{\frac{\alpha}{2}} \mathcal{F}_{L / R, \lambda} f \in L^{2}\left(\mathbb{R}^{n} ; \mathbb{H}\right) .
$$

For $n=1$, (i) and (ii) are equivalent.

We now collect the basic properties of the Sobolev spaces:

Lemma 2.3. Let $\alpha \geq 0, f \in H^{\alpha}\left(\mathbb{R}^{n} ; \mathbb{H}\right)$ and $a, b \in \mathbb{H}$. Then,

(i) $f(-\cdot) \in H^{\alpha}\left(\mathbb{R}^{n} ; \mathbb{H}\right)$ and $\|f\|=\|f(-\cdot)\|$.

(ii) $\bar{f} \in H^{\alpha}\left(\mathbb{R}^{n} ; \mathbb{H}\right)$. In this case $\|f\|=\|\bar{f}\|$.

(iii) (Two-sided homogeneity) $\|a f b\|=|a|\|b \mid\| f \|$.

$H^{\alpha}\left(\mathbb{R}^{n} ; \mathbb{H}\right)$ is left- and right- $\mathbb{H}$-linear.

Moreover, $f \in H^{\alpha}\left(\mathbb{R}^{n} ; \mathbb{H}\right)$ if and only if the four functions $\mathrm{S}(f), \operatorname{Im}_{i}(f)$, $\operatorname{Im}_{j}(f), \operatorname{Im}_{k}(f) \in H^{\alpha}\left(\mathbb{R}^{n} ; \mathbb{H}\right)$. In this case also $\mathrm{V}(f) \in H^{\alpha}\left(\mathbb{R}^{n} ; \mathbb{H}\right)$.

Proof. Apply the equalities from Lemma 1.6. Part (iii) is a consequence of the left- resp. right- $\mathbb{H}$-linearity of $\mathcal{F}_{R}$ resp. $\mathcal{F}_{L}$.

To transfer well-known results for complex Sobolev-spaces to the quaternionic ones, we note the following isomorphism:

\section{Real version.}

$H^{\alpha}\left(\mathbb{R}^{n} ; \mathbb{H}\right) \cong H^{\alpha}\left(\mathbb{R}^{n} ; \mathbb{R}\right) \times H^{\alpha}\left(\mathbb{R}^{n} ; \mathbb{R}\right) \times H^{\alpha}\left(\mathbb{R}^{n} ; \mathbb{R}\right) \times H^{\alpha}\left(\mathbb{R}^{n} ; \mathbb{R}\right)$

as $\mathbb{R}$-vector spaces by virtue of the homeomorphic isomorphism

$$
f \mapsto\left(\mathrm{S}(f), \operatorname{Im}_{i}(f), \operatorname{Im}_{j}(f), \operatorname{Im}_{k}(f)\right) .
$$




\section{Complex version.}

$$
H^{\alpha}\left(\mathbb{R}^{n} ; \mathbb{H}\right) \cong H^{\alpha}\left(\mathbb{R}^{n} ; \mathbb{C}\right) \times H^{\alpha}\left(\mathbb{R}^{n} ; \mathbb{C}\right)
$$

as $\mathbb{C}$-vector spaces by virtue of the homeomorphic isomorphism

$$
f \mapsto \frac{1}{2}(f-i f i,-f j-i f k) .
$$

Theorem 2.4. $\quad$ (i) (Sobolev embedding) Let $\alpha>\ell+\frac{n}{2}, \ell \in \mathbb{N}_{0}$. Then

$$
i d: H^{\alpha}\left(\mathbb{R}^{n} ; \mathbb{H}\right) \hookrightarrow \mathcal{C}^{\ell}\left(\mathbb{R}^{n} ; \mathbb{H}\right) .
$$

(ii) $\mathcal{S}\left(\mathbb{R}^{n} ; \mathbb{H}\right)$ is dense in $H^{\alpha}\left(\mathbb{R}^{n} ; \mathbb{H}\right)$.

(iii) Let $f \in L^{2}\left(\mathbb{R}^{n} ; \mathbb{H}\right)$. Then,

$$
f \in H^{\alpha}\left(\mathbb{R}^{n} ; \mathbb{H}\right) \Leftrightarrow \int_{\mathbb{R}^{n}}|\omega|^{2 \alpha}|\mathcal{F} f(\omega)|^{2} d \omega<\infty
$$

where $\mathcal{F}$ is any of the Fourier transforms $\mathcal{F}_{L}, \mathcal{F}_{R}, \mathcal{F}_{S}$ and the respective unit quaternion can be chosen arbitrarily.

Proof. Direct consequence of the results for $\mathbb{C}$-Sobolev spaces and the above mentioned isomorphism.

\section{Frame Lifting and Partial Ridges}

Let $\beta=\left(\beta_{1}, \ldots, \beta_{n}\right) \in \mathbb{R}_{>0}^{n}$ be a multi-index and $\alpha \geq|\beta|$. For $f \in H^{\alpha}\left(\mathbb{R}^{n} ; \mathbb{H}\right)$ we consider the fractional differential operator

$$
\mathcal{D}^{\beta} f:=\mathcal{F}^{-1}\left(\mathcal{F} f(\cdot)|\cdot|^{\beta}\right) \text {. }
$$

The multi-index notation here is to be $\operatorname{read}$ as $|\beta|:=\sum_{\ell=1}^{n} \beta_{\ell}$ and $|\omega|^{\beta}:=$ $\prod_{\ell=1}^{n}\left|\omega_{\ell}\right|^{\beta_{\ell}}$.

The Fourier transform is set to $\mathcal{F}:=\mathcal{F}_{R}$ and will be set as a convention for the remaining sections however similar results can be formulated for the other versions, too.

Note that $\mathcal{D}^{\beta} f \in H^{\alpha-|\beta|}\left(\mathbb{R}^{n} ; \mathbb{H}\right)$. For $n=1$ we have the equivalence $f \in H^{\alpha}(\mathbb{R} ; \mathbb{H})$ if and only if $\mathcal{D}^{\beta} f \in H^{\alpha-\beta}(\mathbb{R} ; \mathbb{H})$.

The idea now is to consider partial ridges, i.e., ridges over a proper subspace of $\mathbb{R}^{n}$. When doing so, we need an adaption of the Radon transform with respect to these partial ridges.

Definition 3.1. (Multi Radon transform) Let $n, m, n_{1}, \ldots, n_{m} \in \mathbb{N}$ such that $n_{1}+\cdots+n_{m}=n$. Suppose $u_{1} \in \mathbb{S}^{n_{1}-1}, \ldots, u_{m} \in \mathbb{S}^{n_{m}-1}$.

Then the multi Radon transform for a function $f \in L^{1}\left(\mathbb{R}^{n} ; \mathbb{H}\right)$ is defined as

$$
\begin{gathered}
R_{\left(u_{\ell}\right)_{1 \leq \ell \leq m}}: L^{1}\left(\mathbb{R}^{n} ; \mathbb{H}\right) \rightarrow L^{1}\left(\mathbb{R}^{m} ; \mathbb{H}\right) \\
R_{\left(u_{\ell}\right)_{1 \leq \ell \leq m}} f(s):=\int_{u_{1}^{T} v_{1}=s_{1}} \cdots \int_{u_{m}^{T} v_{m}=s_{m}} f\left(v_{1}, \ldots, v_{m}\right) d \sigma\left(v_{m}\right) \cdots d \sigma\left(v_{1}\right) .
\end{gathered}
$$


Here, without loss of generality, we assume that successive coordinates are grouped together. In the following, we will use a shorthand notation for the right-hand integral:

$$
\begin{aligned}
& \iint_{u_{\ell}^{T} v_{\ell}=s_{\ell}} f(x) d \sigma\left(v_{\ell}\right) \\
& \quad:=\int_{u_{1}^{T} v_{1}=s_{1}} \cdots \int_{u_{m}^{T} v_{m}=s_{m}} f\left(v_{1}, \ldots, v_{m}\right) d \sigma\left(v_{m}\right) \cdots d \sigma\left(v_{1}\right),
\end{aligned}
$$

where $x=\left(x_{1}, \ldots, x_{n}\right) \in \mathbb{R}^{n}$, and $v_{1}:=\left(x_{1}, \ldots, x_{n_{1}}\right), v_{2}:=\left(x_{n_{1}+1}, \ldots\right.$, $\left.x_{n_{1}+n_{2}}\right), \ldots, v_{m}:=\left(x_{n-n_{m}+1}, \ldots, x_{n}\right)$ are the partial coordinate vectors.

From now on until the end of this section the setting of Definition 3.1 will be a general assumption and will not be mentioned explicitly anymore.

The following lemma shows that the multi Radon transform maps to $L^{1}\left(\mathbb{R}^{m} ; \mathbb{H}\right)$. Thus the multi Radon integral converges and is well-defined.

Lemma 3.2. For $f \in L^{1}\left(\mathbb{R}^{n} ; \mathbb{H}\right)$,

$$
R_{\left(u_{\ell}\right)_{1 \leq \ell \leq m}} f \in L^{1}\left(\mathbb{R}^{m} ; \mathbb{H}\right) .
$$

Proof.

$$
\begin{aligned}
\int_{\mathbb{R}^{m}}\left|R_{\left(u_{\ell}\right)_{1 \leq \ell \leq m}} f\left(s_{1}, \ldots, s_{m}\right)\right| d\left(s_{1}, \ldots, s_{m}\right) \\
=\int_{\mathbb{R}^{m}}\left|\iint_{u_{\ell}^{T} v_{\ell}=s_{\ell}} f(x) d \sigma\left(v_{\ell}\right)\right| d\left(s_{1}, \ldots, s_{m}\right) \\
\leq \int_{\mathbb{R}^{m}} \iint_{u_{\ell}^{T} v_{\ell}=s_{\ell}}|f(x)| d \sigma\left(v_{\ell}\right) d\left(s_{1}, \ldots, s_{m}\right) \\
=\int_{\mathbb{R}} \int_{u_{1}^{T} v_{1}=s_{1}} \cdots \int_{\mathbb{R}} \int_{u_{m}^{T} v_{m}=s_{m}}\left|f\left(v_{1}, \ldots, v_{m}\right)\right| d \sigma\left(v_{m}\right) d s_{m} \cdots d \sigma\left(v_{1}\right) d s_{1} \\
=\int_{\mathbb{R}^{n_{1}}} \cdots \int_{\mathbb{R}^{n_{m}}}\left|f\left(v_{1}, \ldots, v_{m}\right)\right| d v_{m} \cdots d v_{1}=\|f\|_{1},
\end{aligned}
$$

where we applied the Theorems of Fubini and Tonelli.

The next lemma collects a few properties of the multi Radon transform. All of them are well known from the ordinary Radon transform and are a direct generalization of them. Similar to the usual Radon case a Fourier slice formula holds. In some sense this is an iterated version of the Radon Fourier slice formula.

Lemma 3.3. (Properties of the multi Radon transform) Let $f, g \in L^{1}\left(\mathbb{R}^{n} ; \mathbb{H}\right)$ and $a \in \mathbb{H}$.

(i) (Linearity)

$$
R_{\left(u_{\ell}\right)_{1 \leq \ell \leq m}}(a f+g)=a R_{\left(u_{\ell}\right)_{1 \leq \ell \leq m}} f+R_{\left(u_{\ell}\right)_{1 \leq \ell \leq m}} g .
$$


(ii) (Symmetry property) Let $\lambda \in\{1, \ldots, m\}$. Let $\tilde{u}_{\lambda}=-u_{\lambda}$, and $\tilde{u}_{\ell}=u_{\ell}$ whenever $\ell \neq \lambda$. Then

$$
\begin{aligned}
& R_{\left(\tilde{u}_{\ell}\right)_{1 \leq \ell \leq m}} f\left(s_{1}, \ldots, s_{\lambda-1},-s_{\lambda}, s_{\lambda+1}, \ldots, s_{m}\right) \\
& \quad=R_{\left(u_{\ell}\right)_{1 \leq \ell \leq m}} f\left(s_{1}, \ldots, s_{\lambda-1}, s_{\lambda}, s_{\lambda+1}, \ldots, s_{m}\right) .
\end{aligned}
$$

(iii) (Iterated version of the Fourier Slice Theorem) Let $\eta=\left(\eta_{1}, \ldots, \eta_{m}\right) \in$ $\mathbb{R}^{m}$. Then,

$$
\mathcal{F}\left(R_{\left(u_{\ell}\right)_{1 \leq \ell \leq m}} f\right)\left(\eta_{1}, \ldots, \eta_{m}\right)=\mathcal{F} f\left(\eta_{1} u_{1}, \ldots, \eta_{m} u_{m}\right) .
$$

Proof. (i) and (ii) can be derived by straight-forward calculations. For the proof of (iii), we consider

$$
\begin{aligned}
\mathcal{F} & \left(R_{\left(u_{\ell}\right)_{1 \leq \ell \leq m}} f\right)\left(\eta_{1}, \ldots, \eta_{m}\right) \\
& =\int_{\mathbb{R}^{m}} R_{\left(u_{\ell}\right)_{1 \leq \ell \leq m}} f(s) e^{-2 \pi \mu \eta^{T} s} d s \\
& =\int_{\mathbb{R}^{m}}\left(\iint_{u_{\ell}^{T} v_{\ell}=s_{\ell}} f(x) d \sigma\left(v_{\ell}\right)\right) e^{-2 \pi \mu \eta^{T} s} d s \\
& =\int_{\mathbb{R}} \int_{u_{1}^{T} v_{1}=s_{1}} \cdots \int_{\mathbb{R}} \int_{u_{m}^{T} v_{m}=s_{m}} f\left(v_{1}, \ldots, v_{m}\right) e^{-2 \pi \mu \eta^{T} s} d \sigma\left(v_{m}\right) d s_{m} \cdots d \sigma\left(v_{1}\right) d s_{1} .
\end{aligned}
$$

Now we use the fact that

$$
e^{-2 \pi \mu \eta^{T} s}=e^{-2 \pi \mu \eta_{1} s_{1}} \cdots e^{-2 \pi \mu \eta_{m} s_{m}}
$$

apply the "ordinary" Radon transform, see e.g. [29, Theorem II.1.1].

The next theorem shows a close relation between fractional differential operator $\mathcal{D}^{\beta}$, the multi Radon transform and $L^{2}\left(\mathbb{R}^{m} ; \mathbb{H}\right)$. This result will be needed later on for the proof of the lifting theorem.

Theorem 3.4. Let $m, n \in \mathbb{N}$ and $n_{1}+\cdots+n_{m}=n$. Let $u_{\ell} \in \mathbb{S}^{n_{\ell}-1}$ for $1 \leq \ell \leq$ m. Denote $\beta=\left(\frac{n_{1}-1}{2}, \ldots, \frac{n_{m}-1}{2}\right)$ a multi-index. Suppose $f \in L^{1}\left(\mathbb{R}^{n} ; \mathbb{H}\right) \cap$ $L^{2}\left(\mathbb{R}^{n} ; \mathbb{H}\right)$. Then the following two assertions hold true:

(i)

$$
\mathcal{D}^{\beta}\left(R_{\left(u_{\ell}\right)_{1 \leq \ell \leq m}} f\right) \in L^{2}\left(\mathbb{R}^{m} ; \mathbb{H}\right)
$$

(ii)

$$
\text { for almost every } u=\left(u_{1}, \ldots, u_{m}\right) \text {. }
$$

$$
2^{m}\|f\|_{2}^{2}=\int_{\mathbb{S}^{n_{1}-1}} \cdots \int_{\mathbb{S}^{n} m-1}\left\|\mathcal{D}^{\beta}\left(R_{\left(u_{\ell}\right)_{1 \leq \ell \leq m}} f\right)\right\|^{2} d u_{m} \cdots d u_{1} .
$$

Proof. The proof uses elements of [17, Lemma 2] and generalizes them. As we suppose $f \in L^{1}\left(\mathbb{R}^{n} ; \mathbb{H}\right)$, the multi Radon transform is well defined. Now we consider the $L^{2}$-topology:

$$
\begin{aligned}
\|f\|_{2}^{2} & =\|\mathcal{F} f\|_{2}^{2}=\int_{\mathbb{R}^{n_{1}}} \cdots \int_{\mathbb{R}^{n_{m}}}\left|\mathcal{F} f\left(\omega_{1}, \ldots, \omega_{m}\right)\right|^{2} d \omega \\
& =\left.\left.\int_{\mathbb{S}^{n_{1}-1}} \int_{0}^{\infty} \cdots \int_{\mathbb{S}^{n_{m}-1}} \int_{0}^{\infty}\left|\mathcal{F} f\left(\eta_{1} u_{1}, \ldots, \eta_{m} u_{m}\right)\right| \eta\right|^{\beta}\right|^{2} d \eta_{m} d u_{m} \cdots d \eta_{1} d u_{1}
\end{aligned}
$$




$$
\begin{aligned}
= & \left.\left.\int_{\mathbb{S}^{n_{1}-1}} \int_{0}^{\infty} \cdots \int_{\mathbb{S}^{n_{m}-1}} \int_{0}^{\infty}\left|\mathcal{F}\left(R_{\left(u_{\ell}\right)_{1 \leq \ell \leq m}} f\right)\left(\eta_{1}, \ldots, \eta_{m}\right)\right| \eta\right|^{\beta}\right|^{2} \times \\
& d \eta_{m} d u_{m} \cdots d \eta_{1} d u_{1},
\end{aligned}
$$

applied the Fourier slice theorem. Now, with the Theorems of Fubini and Tonelli and by applying symmetry properties,

$$
\begin{aligned}
\| f & \|_{2}^{2}=\left.\left.\int_{\mathbb{S}^{n_{1}-1}} \cdots \int_{\mathbb{S}^{n_{m}-1}} \int_{0}^{\infty} \cdots \int_{0}^{\infty}\left|\mathcal{F}\left(R_{\left(u_{\ell}\right)_{1 \leq \ell \leq m}} f\right)\left(\eta_{1}, \ldots, \eta_{m}\right)\right| \eta\right|^{\beta}\right|^{2} d \eta_{m} \cdots d \eta_{1} d u_{m} \cdots d u_{1} \\
& =\left.\left.\frac{1}{2^{m}} \int_{\mathbb{S}^{n_{1}-1}} \cdots \int_{\mathbb{S}^{n_{m}-1}} \int_{\mathbb{R}} \cdots \int_{\mathbb{R}}\left|\mathcal{F}\left(R_{\left(u_{\ell}\right)_{1 \leq \ell \leq m}} f\right)\left(\eta_{1}, \ldots, \eta_{m}\right)\right| \eta\right|^{\beta}\right|^{2} d \eta_{m} \cdots d \eta_{1} d u_{m} \cdots d u_{1} \\
& =\frac{1}{2^{m}} \int_{\mathbb{S}^{n_{1}-1}} \cdots \int_{\mathbb{S}^{n_{m}-1}} \int_{\mathbb{R}^{m}}\left|\mathcal{D}^{\beta}\left(R_{\left(u_{\ell}\right)_{1 \leq \ell \leq m}} f\right)(s)\right|^{2} d s d u_{m} \cdots d u_{1} \\
& =\frac{1}{2^{m}} \int_{\mathbb{S}^{n_{1}-1}} \cdots \int_{\mathbb{S}^{n_{m}-1}}\left\|\mathcal{D}^{\beta}\left(R_{\left(u_{\ell}\right)_{1 \leq \ell \leq m}} f\right)\right\|^{2} d u_{m} \cdots d u_{1} .
\end{aligned}
$$

Hence, $\mathcal{D}^{\beta}\left(R_{\left(u_{\ell}\right)_{1 \leq \ell \leq m}} f\right) \in L^{2}\left(\mathbb{R}^{m} ; \mathbb{H}\right)$ for almost all $u=\left(u_{1}, \ldots, u_{m}\right)$.

From the previous theorem we easily deduce a $L^{2}\left(\mathbb{R}^{m} ; \mathbb{H}\right)$ condition for almost all directions.

Corollary 3.5. Suppose $f \in L^{1}\left(\mathbb{R}^{n} ; \mathbb{H}\right) \cap L^{2}\left(\mathbb{R}^{n} ; \mathbb{H}\right)$. Then $R_{\left(u_{\ell}\right)_{1 \leq \ell \leq m}} f \in$ $L^{1}\left(\mathbb{R}^{m} ; \mathbb{H}\right) \cap L^{2}\left(\mathbb{R}^{m} ; \mathbb{H}\right)$ for almost all $u_{\ell} \in \mathbb{S}^{n_{\ell}-1}$ where $1 \leq \ell \leq m$.

Proof. We have $R_{\left(u_{\ell}\right)_{1<\ell<m}} f \in L^{1}\left(\mathbb{R}^{m} ; \mathbb{H}\right)$. Applying Theorem 3.4 yields for the multi-index $\beta=\left(\frac{n_{1}-1}{2}, \ldots, \frac{n_{m}-1}{2}\right)$ :

$$
\mathcal{D}^{\beta}\left(R_{\left(u_{\ell}\right)_{1 \leq \ell \leq m}} f\right) \in L^{2}\left(\mathbb{R}^{m} ; \mathbb{H}\right)
$$

for almost all $u_{\ell} \in \mathbb{S}^{n_{\ell}-1}$ where $1 \leq \ell \leq m$. Then-as the preimage is more regular in terms of Sobolev-regularity - we deduce $R_{\left(u_{\ell}\right)_{1 \leq \ell \leq m}} f \in H^{\alpha}\left(\mathbb{R}^{m} ; \mathbb{H}\right)$ for some $\alpha \geq 0$.

Thus $R_{\left(u_{\ell}\right)_{1 \leq \ell \leq m}} f \in L^{1}\left(\mathbb{R}^{m} ; \mathbb{H}\right) \cap L^{2}\left(\mathbb{R}^{m} ; \mathbb{H}\right)$.

\subsection{Multivariate Ridge Construction}

Definition 3.6. (Multivariate ridge construction) Let $n, m, n_{1}, \ldots, n_{m} \in \mathbb{N}$ such that $n_{1}+\cdots+n_{m}=n$. Suppose $u_{1} \in \mathbb{S}^{n_{1}-1}, \ldots, u_{m} \in \mathbb{S}^{n_{m}-1}$.

Then the multivariate ridge construction for a function $g: \mathbb{R}^{m} \rightarrow \mathbb{H}$ is defined as

$$
g_{\left(u_{\ell}\right)_{1 \leq \ell \leq m}}: \mathbb{R}^{n} \rightarrow \mathbb{H}, g_{\left(u_{\ell}\right)_{1 \leq \ell \leq m}}(x):=g\left(u_{1}^{T} v_{1}, \ldots, u_{m}^{T} v_{m}\right)
$$

where $x=\left(x_{1}, \ldots, x_{n}\right)$, and $v_{1}=\left(x_{1}, \ldots, x_{n_{1}}\right), v_{2}=\left(x_{n_{1}+1}, \ldots, x_{n_{1}+n_{2}}\right)$, $\ldots, v_{m}=\left(x_{n-n_{m}+1}, \ldots, x_{n}\right)$.

Here again, without loss of generality, we assumed that only successive coordinates are grouped together.

Lemma 3.7. Let $n>m$. Suppose $\left(u_{\ell}\right)_{1 \leq \ell \leq m}$ as in Definition 3.6.

(i) Let $k \in \mathbb{N}, g \in \mathcal{C}^{k}\left(\mathbb{R}^{m} ; \mathbb{H}\right)$ then $g_{\left(u_{\ell}\right)_{1<\ell<m}} \in \mathcal{C}^{k}\left(\mathbb{R}^{n} ; \mathbb{H}\right)$.

(ii) Let $g \in L^{\infty}\left(\mathbb{R}^{m} ; \mathbb{H}\right)$ then $g_{\left(u_{\ell}\right)_{1<\ell<m}} \in L^{\infty}\left(\mathbb{R}^{n} ; \mathbb{H}\right)$.

Proof. This follows from the smoothness of the ridge construction $\mathbb{R}^{n} \rightarrow \mathbb{R}^{m}$, $x \mapsto\left(u_{1}^{T} v_{1}, \ldots, u_{m}^{T} v_{m}\right)$ where $x$ and $v_{1}, \ldots, v_{m}$ as in Definition 3.6. 
Note that embedding results may not hold in the $L^{p}$-topology: Let $1 \leq$ $p<\infty$, and $g \in L^{p}\left(\mathbb{R}^{m} ; \mathbb{H}\right)$. Then in general $g_{\left(u_{\ell}\right)_{1 \leq \ell \leq m}} \notin L^{p}\left(\mathbb{R}^{n} ; \mathbb{H}\right)$. For example, consider $g(x):=e^{-x^{2}}, g \in \mathcal{S}(\mathbb{R} ; \mathbb{H})$. By density of $\mathcal{S}(\mathbb{R} ; \mathbb{H})$ in $L^{p}(\mathbb{R} ; \mathbb{H})$ it immediately follows that $g \in L^{p}(\mathbb{R} ; \mathbb{H})$. Now we consider $m=1$, $n=2, u=\left(\begin{array}{l}1 \\ 0\end{array}\right)$. Then $g_{u}(x, y)=e^{-x^{2}}$. This function does not decay in $y$-direction, and hence it is not contained in any $L^{p}\left(\mathbb{R}^{n} ; \mathbb{H}\right)$.

The following lemma shows a close relation between multivariate ridge functions and the multi Radon transform. The idea of the proof is based on [17, Lemma 1] and [10, Lemma 1.3].

For functions $f, g: \mathbb{R}^{\ell} \rightarrow \mathbb{H}, \ell \in \mathbb{N}$, we denote

$$
\langle f, g\rangle=\int_{\mathbb{R}^{\ell}} f(x) \overline{g(x)} d x,
$$

whenever the right hand side converges.

Lemma 3.8. Let $n \geq m, m, n \in \mathbb{N}$. Suppose $f \in L^{1}\left(\mathbb{R}^{n} ; \mathbb{H}\right), g \in L^{\infty}\left(\mathbb{R}^{m} ; \mathbb{H}\right)$. Then

$$
\left\langle f, g_{\left(u_{\ell}\right)_{1 \leq \ell \leq m}}\right\rangle=\left\langle R_{\left(u_{\ell}\right)_{1 \leq \ell \leq m}} f, g\right\rangle,
$$

where the first $\langle\cdot, \cdot\rangle$ considers function on $\mathbb{R}^{m}$, and the second on $\mathbb{R}^{m}$.

Proof. The expression on the left side is well-defined, because it is finite:

$$
\begin{aligned}
& \int_{\mathbb{R}^{n}}\left|f\left(v_{1}, \ldots, v_{m}\right) \overline{g\left(u_{1}^{T} v_{1}, \ldots, u_{m}^{T} v_{m}\right)}\right| d\left(v_{1}, \ldots, v_{m}\right) \\
& \quad \leq\|g\|_{L^{\infty}\left(\mathbb{R}^{m} ; \mathbb{H}\right)} \int_{\mathbb{R}^{n}}\left|f\left(v_{1}, \ldots, v_{m}\right)\right| d\left(v_{1}, \ldots, v_{m}\right) \\
& \quad=\|g\|_{L^{\infty}\left(\mathbb{R}^{m} ; \mathbb{H}\right)}\|f\|_{L^{1}\left(\mathbb{R}^{n} ; \mathbb{C}\right)}<\infty .
\end{aligned}
$$

We apply Fubini's Theorem several times and deduce:

$$
\begin{aligned}
\left\langle f, g_{\left(u_{\ell}\right)_{1 \leq \ell \leq m}}\right\rangle & \\
= & \int_{\mathbb{R}^{n_{1}}} \cdots \int_{\mathbb{R}^{n_{m}}} f\left(v_{1}, \ldots, v_{m}\right) \overline{g\left(u_{1}^{T} v_{1}, \ldots, u_{m}^{T} v_{m}\right)} d v_{m} \ldots \ldots d v_{1} \\
= & \int_{\mathbb{R}} \int_{u_{1}^{T} v_{1}=s_{1}} \cdots \int_{\mathbb{R}} \int_{u_{m}^{T} v_{m}=s_{m}} f\left(v_{1}, \ldots, v_{m}\right) \overline{g\left(u_{1}^{T} v_{1}, \ldots, u_{m}^{T} v_{m}\right)} \times \\
& d \sigma\left(v_{m}\right) d s_{m} \cdots d \sigma\left(v_{1}\right) d s_{1} \\
= & \int_{\mathbb{R}^{m}} \int_{u_{1}^{T} v_{1}=s_{1}} \cdots \int_{u_{m}^{T} v_{m}=s_{m}} f\left(v_{1}, \ldots, v_{m}\right) \overline{g\left(s_{1}, \ldots, s_{m}\right)} \times \\
& d \sigma\left(v_{m}\right) \cdots d \sigma\left(v_{1}\right) d\left(s_{1}, \ldots, s_{m}\right) \\
= & \int_{\mathbb{R}^{m}} R_{\left(u_{\ell}\right)_{1 \leq \ell \leq m}} f(s) \overline{g(s)} d s=\left\langle R_{\left(u_{\ell}\right)_{1 \leq \ell \leq m}} f, g\right\rangle .
\end{aligned}
$$

\subsection{Frame Lifting}

The following theorem is the main theorem: the extension of the frame lifting idea of [17] and [10] in terms of quaternions and multivariate frames. 
Theorem 3.9. Let $m, n, n_{1}, \ldots, n_{m} \in \mathbb{N}$ such that $\sum_{\ell=1}^{m} n_{\ell}=n$. Moreover, let $M$ be a measurable set. Let $\left\{f_{\kappa}\right\}_{\kappa \in M},\left\{g_{\kappa}\right\}_{\kappa \in M} \subset H^{\frac{1}{2}(n-m)}\left(\mathbb{R}^{m} ; \mathbb{H}\right)$ be a dual $L^{2}\left(\mathbb{R}^{m} ; \mathbb{H}\right)$ continuous frame pair with frame bounds $A, B>0$. Define $F_{\kappa}:=\mathcal{D}^{\beta} f_{\kappa}, G_{\kappa}:=\mathcal{D}^{\beta} g_{\kappa}$ for every $\kappa \in M$ where $\beta=\left(\frac{n_{1}-1}{2}, \ldots, \frac{n_{m}-1}{2}\right)$ is a multi-index. Suppose that $F_{\kappa}, G_{\kappa} \in L^{\infty}\left(\mathbb{R}^{m} ; \mathbb{H}\right)$.

Then for $f \in L^{1}\left(\mathbb{R}^{n} ; \mathbb{H}\right) \cap L^{2}\left(\mathbb{R}^{n} ; \mathbb{H}\right)$ the following two assertions hold true:

$$
f=\frac{1}{2^{m}} \int_{\mathbb{S}^{n_{1}-1}} \cdots \int_{\mathbb{S}^{n} m-1} \int_{M}\left\langle f, G_{\kappa,\left(u_{\ell}\right)_{1 \leq \ell \leq m}}\right\rangle F_{\kappa,\left(u_{\ell}\right)_{1 \leq \ell \leq m}} d \kappa d u_{m} \cdots d u_{1} .
$$

$$
2^{m} A\|f\|_{2}^{2} \leq \int_{\mathbb{S}^{n_{1}-1}} \cdots \int_{\mathbb{S}^{n} m-1} \int_{M}\left|\left\langle f, G_{\kappa,\left(u_{\ell}\right)_{1 \leq \ell \leq m}}\right\rangle\right|^{2} d \kappa d u_{m} \cdots d u_{1} \leq 2^{m} B\|f\|_{2}^{2} .
$$

The idea of the proof is based on the proofs of [17] and [10], and fills a gap in the proof in [10] with regard to Sobolev spaces.

Note that the families

$$
\left(F_{\kappa,\left(u_{\ell}\right)_{1 \leq \ell \leq m}}\right)_{\kappa \in M, u \in \mathbb{S}^{n_{1}-1} \times \cdots \times \mathbb{S}^{n_{m}-1}},\left(G_{\kappa,\left(u_{\ell}\right)_{1 \leq \ell \leq m}}\right)_{\kappa \in M, u \in \mathbb{S}^{n_{1}-1} \times \cdots \times \mathbb{S}^{n_{m}-1}}
$$

allow for a decomposition as continuous frames do. However, they are not continuous frames themselves, as they are not in $L^{2}\left(\mathbb{R}^{n} ; \mathbb{H}\right)$.

Proof. Since $|\beta|=\sum_{i=1}^{m} \frac{n_{i}-1}{2}=\frac{1}{2}(n-m)$ the expressions $\mathcal{D}^{\beta} f_{\kappa}, \mathcal{D}^{\beta} g_{\kappa}$ are well defined for all $\kappa \in M$ because of the definition of $\mathcal{D}$.

Lemma 3.7 implies that $G_{\kappa,\left(u_{\ell}\right)_{1<\ell<m}} \in L^{\infty}\left(\mathbb{R}^{n} ; \mathbb{H}\right)$. Hence the preconditions of Lemma 3.8 are fulfilled and we can rewrite:

$$
\begin{aligned}
& \left\langle f, G_{\kappa,\left(u_{\ell}\right)_{1 \leq \ell \leq m}}\right\rangle=\left\langle R_{\left(u_{\ell}\right)_{1 \leq \ell \leq m}} f, G_{\kappa}\right\rangle=\left\langle\mathcal{F}\left(R_{\left(u_{\ell}\right)_{1 \leq \ell \leq m}} f\right), \mathcal{F}\left(g_{\kappa}\right)|\cdot|^{\beta}\right\rangle \\
& \quad=\left\langle\mathcal{F}\left(R_{\left(u_{\ell}\right)_{1 \leq \ell \leq m}} f\right)|\cdot|^{\beta}, \mathcal{F}\left(g_{\kappa}\right)\right\rangle .
\end{aligned}
$$

Now, we prove (i):

For $x=\left(x_{1}, \ldots, x_{n}\right)$ and

$$
v_{1}=\left(x_{1}, \ldots, x_{n_{1}}\right), v_{2}=\left(x_{n_{1}+1}, \ldots, x_{n_{1}+n_{2}}\right), \ldots, v_{m}=\left(x_{n-n_{m}+1}, \ldots, x_{n}\right)
$$

similar to Definition 3.6 the second frame of the pair is rewritten as

$$
\begin{aligned}
F_{\kappa,\left(u_{\ell}\right)_{1 \leq \ell \leq m}}(x) & =F_{\kappa}\left(v_{1}^{T} u_{1}, \ldots, v_{m}^{T} u_{m}\right)=\mathcal{D}^{\beta}\left(f_{\kappa}\right)\left(v_{1}^{T} u_{1}, \ldots, v_{m}^{T} u_{m}\right) \\
& =\mathcal{F}^{-1}\left(\mathcal{F}\left(f_{\kappa}\right)|\cdot|^{\beta}\right)\left(v_{1}^{T} u_{1}, \ldots, v_{m}^{T} u_{m}\right) .
\end{aligned}
$$

We combine the results above:

$$
\begin{aligned}
\frac{1}{2^{m}} & \int_{\mathbb{S}^{n_{1}-1}} \cdots \int_{\mathbb{S}^{n} m-1} \int_{M}\left\langle f, G_{\kappa,\left(u_{\ell}\right)_{1 \leq \ell \leq m}}\right\rangle F_{\kappa,\left(u_{\ell}\right)_{1 \leq \ell \kappa \leq m}}(x) d \kappa d u_{m} \cdots d u_{1} \\
= & \frac{1}{2^{m}} \int_{\mathbb{S}^{n_{1}-1}} \cdots \int_{\mathbb{S}^{n_{m}-1}} \int_{M}\left\langle\mathcal{F}\left(R_{\left(u_{\ell}\right)_{1 \leq \ell \leq m}} f\right)|\cdot|^{\beta}, \mathcal{F}\left(g_{\kappa}\right)\right\rangle \\
& \mathcal{F}^{-1}\left(\mathcal{F}\left(f_{\kappa}\right)|\cdot|^{\beta}\right)\left(v_{1}^{T} u_{1}, \ldots, v_{m}^{T} u_{m}\right) d \kappa d u_{m} \cdots d u_{1} \\
= & \frac{1}{2^{m}} \int_{\mathbb{S}^{n_{1}-1}} \cdots \int_{\mathbb{S}^{n} m-1} \mathcal{F}^{-1}\left(\int_{M}\left\langle\mathcal{F}\left(R_{\left(u_{\ell}\right)_{1 \leq \ell \leq m}} f\right)|\cdot|^{\beta}, \mathcal{F}\left(g_{\kappa}\right)\right\rangle \mathcal{F}\left(f_{\kappa}\right)|\cdot|^{\beta} d \kappa\right)
\end{aligned}
$$




$$
\begin{gathered}
\left(v_{1}^{T} u_{1}, \ldots, v_{m}^{T} u_{m}\right) d u_{m} \cdots d u_{1} \\
=\frac{1}{2^{m}} \int_{\mathbb{S}^{n_{1}-1}} \cdots \int_{\mathbb{S}^{n} m-1} \mathcal{F}^{-1}\left(\int_{M}\left\langle\mathcal{F}\left(R_{\left(u_{\ell}\right)_{1 \leq \ell \leq m}} f\right)|\cdot|^{\beta}, \mathcal{F}\left(g_{\kappa}\right)\right\rangle \mathcal{F}\left(f_{\kappa}\right) d \kappa|\cdot|^{\beta}\right) \\
\quad\left(v_{1}^{T} u_{1}, \ldots, v_{m}^{T} u_{m}\right) d u_{m} \cdots d u_{1}=: A(x) .
\end{gathered}
$$

Now, inside the big parentheses there is a frame decomposition of the function $\mathcal{F}\left(R_{\left(u_{\ell}\right)_{1 \leq \ell \leq m}} f\right)$ multiplied by the factor $|\cdot|^{\beta}$ :

$$
\mathcal{F}\left(R_{\left(u_{\ell}\right)_{1 \leq \ell \leq m}} f\right)|\cdot|^{\beta}=\int_{M}\left\langle\mathcal{F}\left(R_{\left(u_{\ell}\right)_{1 \leq \ell \leq m}} f\right)|\cdot|^{\beta}, \mathcal{F}\left(g_{\kappa}\right)\right\rangle \mathcal{F}\left(f_{\kappa}\right) d \kappa|\cdot|^{\beta} .
$$

Applying the Fourier Slice Theorem 3.3 yields:

$$
\begin{gathered}
A(x)=\frac{1}{2^{m}} \int_{\mathbb{S}^{n_{1}-1}} \cdots \int_{\mathbb{S}^{n} m-1} \mathcal{F}^{-1}\left(\mathcal{F}(f)\left(\cdot{ }_{1} u_{1}, \ldots, \cdot{ }_{m} u_{m}\right)|\cdot|^{2 \beta}\right) \\
\left(v_{1}^{T} u_{1}, \ldots, v_{m}^{T} u_{m}\right) d u_{m} \cdots d u_{1} .
\end{gathered}
$$

Inserting the formula of the inverse Fourier transform over $L^{2}\left(\mathbb{R}^{m} ; \mathbb{H}\right)$ implies

$$
\begin{gathered}
A(x)=\frac{1}{2^{m}} \int_{\mathbb{S}^{n_{1}-1}} \cdots \int_{\mathbb{S}^{n} m-1} \int_{\mathbb{R}^{m}} \mathcal{F}(f)\left(\eta_{1} u_{1}, \ldots, \eta_{m} u_{m}\right)|\cdot|^{2 \beta} \\
e^{2 \pi \mu\left(v_{1}^{T} u_{1}, \ldots, v_{m}^{T} u_{m}\right) \eta} d \eta d u_{m} \cdots d u_{1} .
\end{gathered}
$$

The application of Fubini's Theorem allows for splitting. Afterwards the symmetry properties of $\eta_{i} u_{i}$ are used.

$$
\begin{aligned}
A(x)= & \frac{1}{2^{m}} \int_{\mathbb{S}^{n_{1}-1}} \int_{\mathbb{R}} \ldots \int_{\mathbb{S}^{n_{m}-1}} \int_{\mathbb{R}} \mathcal{F}(f)\left(\eta_{1} u_{1}, \ldots, \eta_{m} u_{m}\right)|\cdot|^{2 \beta} \\
= & \int_{\mathbb{S}^{n_{1}-1}} \int_{0}^{2 \pi \mu\left(v_{1}^{T} u_{1}, \ldots, v_{m}^{T} u_{m}\right) \eta} d \eta_{m} d u_{m} \cdots d \eta_{1} d u_{1} \\
& e^{2 \pi \mu\left(v_{1}^{T} \eta_{1} u_{1}+\cdots+v_{m}^{T} \eta_{m} u_{m}\right)} \int_{0}^{\infty} \mathcal{F}(f)\left(\eta_{1} u_{1}, \ldots, \eta_{m} u_{m}\right)|\cdot|^{2 \beta} \\
= & \int_{\mathbb{R}^{n}} \mathcal{F}(f)\left(y_{1}, \ldots, y_{m}\right) e^{2 \pi \mu\left(v_{1}^{T} y_{1}+\cdots+v_{m}^{T} y_{m}\right)} d y_{m} \cdots d \eta_{1} d u_{1} \\
= & f\left(v_{1}, \ldots, v_{m}\right)=f(x) \quad \text { in the } L^{2} \text {-sense. }
\end{aligned}
$$

To prove part (ii) we reconsider (1):

$$
\left\langle f, G_{\kappa,\left(u_{\ell}\right)_{1 \leq \ell \leq m}}\right\rangle=\left\langle\mathcal{F}\left(R_{\left(u_{\ell}\right)_{1 \leq \ell \leq m}} f\right)|\cdot|^{\beta}, \mathcal{F} g_{\kappa}\right\rangle=\left\langle\mathcal{D}^{\beta}\left(R_{\left(u_{\ell}\right)_{1 \leq \ell \leq m}} f\right), g_{\kappa}\right\rangle \text {. }
$$

Hence,

$$
\int_{M}\left|\left\langle f, G_{\kappa,\left(u_{\ell}\right)_{1 \leq \ell \leq m}}\right\rangle\right| d \kappa=\int_{M}\left|\left\langle\mathcal{D}^{\beta}\left(R_{\left(u_{\ell}\right)_{1 \leq \ell \leq m}} f\right), g_{\kappa}\right\rangle\right| d \kappa
$$

Thus, by the frame property of $\left\{g_{\kappa}\right\}_{\kappa \in M}$, we deduce

$$
A\left\|\mathcal{D}^{\beta}\left(R_{\left(u_{\ell}\right)_{1 \leq \ell \leq m}} f\right)\right\|_{2}^{2} \leq \int_{M}\left|\left\langle f, G_{\kappa,\left(u_{\ell}\right)_{1 \leq \ell \leq m}}\right\rangle\right| d \kappa \leq B\left\|\mathcal{D}^{\beta}\left(R_{\left(u_{\ell}\right)_{1 \leq \ell \leq m}} f\right)\right\|_{2}^{2} .
$$

From Theorem 3.4 it is known that

$$
2^{m}\|f\|_{2}^{2}=\int_{\mathbb{S}^{n_{1}-1}} \cdots \int_{\mathbb{S}^{n} m-1}\left\|\mathcal{D}^{\beta}\left(R_{\left(u_{\ell}\right)_{1 \leq \ell \leq m}} f\right)\right\|_{2}^{2} d u_{m} \cdots d u_{1} .
$$


Thus integrating over the spheres yields the desired result.

A natural question is whether this way of generalizing the frame decomposition to higher numbers of variables is canonical in a sense that it coincides with the ordinary frame decomposition if $n=m$, i.e., if all $n_{\ell}=1$. Indeed this is the case. Let all $n_{\ell}=1$ for $1 \leq \ell \leq m$ then $\beta=0$. By definition of $\mathcal{D}$ it immediately follows that $F_{\kappa}=\mathcal{D}^{\beta} f_{\kappa}=f_{\kappa}$ and $G_{\kappa}=\mathcal{D}^{\beta} g_{\kappa}=g_{\kappa}$ for all $\kappa \in M$. The spheres collapse to $\mathbb{S}^{n_{\ell}-1}=\{-1,1\}$ for $1 \leq \ell \leq m$. Thus the integral becomes a discrete sum, and the directions $u_{\ell}$ become reflections with respect to the $m$ variables of $f$.

$$
\begin{aligned}
f(x) & =\frac{1}{2^{m}} \int_{\mathbb{S}^{n_{1}-1}} \cdots \int_{\mathbb{S}^{n} m-1} \int_{M}\left\langle f, G_{\kappa,\left(u_{\ell}\right)_{1 \leq \ell \leq m}}\right\rangle F_{\kappa,\left(u_{\ell}\right)_{1 \leq \ell \leq m}}(x) d \kappa d u_{m} \cdots d u_{1} \\
& =\frac{1}{2^{m}} \sum_{\left(u_{\ell}\right)_{1 \leq \ell \leq m} \in\{-1,1\}^{m}} \int_{M}\left\langle f, g_{\kappa}\left(u_{1} \cdot \ldots, u_{m} \cdot\right)\right\rangle f_{\kappa}\left(u_{1} x_{1}, u_{m} x_{m}\right) d \kappa .
\end{aligned}
$$

The dot-notation $u_{1} \cdot, u_{2} \cdot, \ldots$ indicates that there are arguments to be inserted. The arguments appear explicitely when the inner product is evaluated. E.g. $h\left(u_{1} \cdot, u_{2} \cdot, \ldots\right): \mathbb{R}^{2} \rightarrow \mathbb{R},\left(x_{1}, x_{2}\right) \mapsto h\left(u_{1} x_{1}, u_{2} x_{2}\right)$.

Now, in the upper formula, there are just $2^{m}$ frame decompositions and synthesis with all possible different reflections of the frames, which are again frames themselves.

Hence the constant $2^{m}$ cancels and $f$ is the final result as expected. In this sense this factor is natural and just the discrete analogy of the $m$ integral changes from $\int_{0}^{\infty}$ to $\int_{\mathbb{R}}$ in the proof.

For the inequality

$$
2^{m} A\|f\|_{2}^{2} \leq \int_{\mathbb{S}^{n}{ }^{-1}} \cdots \int_{\mathbb{S}^{n} m-1} \int_{M}\left|\left\langle f, G_{\kappa,\left(u_{\ell}\right)_{1 \leq \ell \leq m}}\right\rangle\right|^{2} d \kappa d u_{m} \cdots d u_{1} \leq 2^{m} B\|f\|_{2}^{2}
$$

similar arguments show equality to

$$
2^{m} A\|f\|_{2}^{2} \leq \sum_{\left(u_{\ell}\right)_{1 \leq \ell \leq m} \in\{-1,1\}^{m}} \int_{M}\left|\left\langle f, g_{\kappa}\left(u_{1} \cdot \ldots, u_{m} \cdot\right)\right\rangle\right|^{2} d \kappa \leq 2^{m} B\|f\|_{2}^{2} .
$$

The reflected frames have of course the same bounds and thus $2^{m}$ can be canceled which yields

$$
A\|f\|_{2}^{2} \leq \int_{M}\left|\left\langle f, g_{\kappa}\right\rangle\right|^{2} d \kappa \leq B\|f\|_{2}^{2}
$$

as desired.

Remark 3.10. The requirements for regularity of Theorem 3.9 are stronger than needed in some cases because not performing a ridge construction in some variables implies that no special regularity is needed for this direction. Thus it suffices to have a suitable degree of regularity in every direction according to the ridge construction. However, we will not pursue this further, here. 
In general, it is not so easy to see whether the image of $\mathcal{D} \beta$ is bounded. Furthermore computing the fractional differential operator for non-even orders in general is very difficult. Luckily, there is a good, sufficient and easy to check for boundedness under mild additional preconditions:

Lemma 3.11. (Bounded range) Let $f \in H^{\alpha}\left(\mathbb{R}^{m} ; \mathbb{H}\right)$ and $\beta \in \mathbb{R}_{>0}^{m}$ multiindex such that $|\beta| \leq \alpha$ holds. Additionally suppose $\mathcal{F}(f)|\cdot|^{\beta} \in L^{1}\left(\mathbb{R}^{m} ; \mathbb{H}\right)$. Then, $\mathcal{D}^{\beta} f \in \mathcal{C}_{0}\left(\mathbb{R}^{m} ; \mathbb{H}\right)$.

Proof. This follows from the Riemann-Lebesgue lemma.

Of course the $L^{1}\left(\mathbb{R}^{m} ; \mathbb{H}\right)$ property is not automatically fulfilled, however it is not a strong requirement either because $\mathcal{F}(f)|\cdot|^{\beta} \in L^{2}\left(\mathbb{R}^{m} ; \mathbb{H}\right)$ has to be fulfilled in any case and the intersection $L^{2}\left(\mathbb{R}^{m} ; \mathbb{H}\right) \cap L^{1}\left(\mathbb{R}^{m} ; \mathbb{H}\right)$ is dense in $L^{2}\left(\mathbb{R}^{m} ; \mathbb{H}\right)$.

From an implementational point of view having a continuous function as a derivative which vanishes at infinity is also a desirable property. On the one hand it increases numerical stability because of continuity and on the other hand the integrals can only be computed over a finite domain which is only possible if the functions vanish.

For $\mathcal{F}(f)|\cdot|^{\beta} \in L^{2}\left(\mathbb{R}^{m} ; \mathbb{H}\right)$ this holds not automatically true as the function $\mathcal{D}^{\beta} f$ in general is neither continuous nor does it vanish.

Lemma 3.12. (Necessary condition for frames) Let $\left\{f_{\kappa}\right\}_{\kappa \in M} \subset L^{2}\left(\mathbb{R}^{m} ; \mathbb{H}\right)$ and let $n \in \mathbb{N}$ be the dimension the frame shall be lifted to. Suppose $\ell \in \mathbb{N}$ is the largest number such that $\ell<\frac{1}{2} n-m$. Then, $\left\{f_{\kappa}\right\}_{\kappa \in M} \subset \mathcal{C}^{\ell} m$.

Proof. Theorem 3.9 has the requirement that the Sobolev regularity of the frames has to be at least $\frac{1}{2}(n-m)$. Now the Sobolev embedding Theorem 2.4 states that $\frac{1}{2}(n-m)>\ell+\frac{m}{2} \Leftrightarrow \ell<\frac{1}{2} n-m$. This proves the claim.

\section{Examples for Lifted Frames}

This section investigates the frame classes wavelets and shearlets for applicability with the lifting procedure in Theorem 3.9. We illustrate the possibilities and boundaries of the introduced theory. Our choice is based on popularity of these frame classes and to illustrate interesting phenomena.

For these two frame classes and many others like Gabor frames or continuous wavelet frames there naturally arise two questions:

1. Does it suffice to show that the generating functions fulfills the requirements for the lifting procedure? Will this imply that this is the case for the whole frame?

2. Do the operations to generate the frame commute with the fractional differential operator $\mathcal{D}^{\beta}$ ? Or is there at least some intertwining property? If this is the case, the structure and therefore the interpretations lift to the generalized setting. As a consequence, the fractional differential operator only needs to be computed for the generating function. 


\subsection{Discrete Wavelets}

Discrete wavelets consist of a tight $L^{2}(\mathbb{R} ; \mathbb{H})$ frame out of translations and dilations of a single generating wavelet $\psi$. This theory is well understood and developed for the real and complex valued case and numerous concrete wavelets, conditions, propositions and applications exist. For further reading consider [9] and [28]. For the quaternion-valued setting some theories have been developed in [2] and [20], but to the best of our knowledge no concrete non-trivial $\mathbb{H}$-valued discrete wavelets have been developed so far. For an overview on recent quaternion wavelet filters see [16].

In this subsection we first define the fundamental wavelet setting. Second, we reconsider the questions from Sect. 1 and develop their answers.

In this case it suffices to show that the wavelet itself fulfills the requirements in order to hold for the whole frame, and that the translation-dilation structure lifts as both operators almost commute with $\mathcal{D}^{\beta}$. These results are finally summarized in a specialized version of the main theorem 3.9 for the discrete wavelet setting. Finally, some examples of concrete wavelets are shown.

As already stated, wavelet systems are generated by translations and dilations of a single generating wavelet.

Let $f \in L^{2}(\mathbb{R} ; \mathbb{H}), a, b, \omega \in \mathbb{R}$ and $\mu \in \mathrm{V}(\mathbb{H})$. Then translation $T^{b}$, dilation $D^{a}$ and modulation $E_{\omega}$ are defined as

$$
\begin{aligned}
T^{b}, D^{a}, E_{\omega}: L^{2}(\mathbb{R} ; \mathbb{H}) \rightarrow L^{2}(\mathbb{R} ; \mathbb{H}), \\
T^{b} f:=f(\cdot-b), \\
D^{a} f:=2^{a / 2} f\left(2^{a} \cdot\right), \\
E_{\omega} f(\cdot):=f(\cdot) e^{2 \pi \mu \omega .} .
\end{aligned}
$$

Note that the order of the function $f$ and the exponential term is important in the quaternionic setting. Similar to the three definitions of the Fourier transform, there are three fundamental types of modulations. A left modulation, a right modulation (introduced in the previous definition) and a sandwich version. Similarly there is more freedom in the choice of the pure unit quaternion compared to the possible $\pm i$ in the complex setting. Each of them corresponds to the respective Fourier transform definition and is compatible to it.

In this section we will restrict ourselves to the case of the modulation from the right and the Fourier transform $\mathcal{F}_{R}$. Similar results can be derived for $\mathcal{F}_{L}$ and $\mathcal{F}_{S}$.

Definition 4.1. (Wavelet system) A function $\psi \in L^{2}(\mathbb{R} ; \mathbb{H})$ is called a wavelet if the system

$$
\left(D^{a} T^{b} \psi\right)_{a, b \in \mathbb{Z}}
$$

is an orthonormal basis for $L^{2}(\mathbb{R} ; \mathbb{H})$.

Translation and dilation are compatible with the $H^{\alpha}(\mathbb{R} ; \mathbb{H})$ setting: The quaternion-valued Sobolev spaces are invariant with respect to translations $T_{b}$ and dilations $D^{a}$ for all $a, b \in \mathbb{R}$. $\mathcal{D}^{\beta}$ intertwines with $D^{a}$ and $T^{b}$. 
Lemma 4.2. Let $f \in H^{\alpha}(\mathbb{R} ; \mathbb{H})$ and $\alpha \geq \beta$. For $a, b \in \mathbb{R}$ :

(i) $\mathcal{D}^{\beta}\left(T^{b} f\right)=T^{b} \mathcal{D}^{\beta} f$.

(ii) $\mathcal{D}^{\beta}\left(D^{a} f\right)=2^{\beta a} D^{a} \mathcal{D}^{\beta} f$.

Proof. Note that the fractional differential operator $\mathcal{D}^{\beta}$ becomes a factor $|\cdot|^{\beta}$ in Fourier domain. Direct calculation gives the result.

In summary this implies that the wavelet structure and thus the interpretation is lifted. Furthermore, the fractional differential operator has to be computed or approximated only once and then can be translated and appropriately scaled.

Finally, to summarize this subsection we formulate the frame lifting theorem 3.9 for the discrete wavelet case.

Theorem 4.3. Let $n \in \mathbb{N}$ and let $\psi \in H^{\frac{n-1}{2}}(\mathbb{R} ; \mathbb{H})$ be a wavelet. Define

$$
\Psi_{a, b}:=2^{a \frac{n-1}{2}} D^{a} T^{b} \mathcal{D}^{\frac{n-1}{2}}(\psi),
$$

and suppose $\Psi_{0,0} \in L^{\infty}(\mathbb{R} ; \mathbb{H})$.

Then for $f \in L^{1}\left(\mathbb{R}^{n} ; \mathbb{H}\right) \cap L^{2}\left(\mathbb{R}^{n} ; \mathbb{H}\right)$,

(i)

$$
f=\frac{1}{2} \int_{\mathbb{S}^{n-1}} \sum_{a, b \in \mathbb{Z}}\left\langle f, \Psi_{a, b, u}\right\rangle \Psi_{a, b, u} d u
$$

(ii)

$$
2\|f\|_{2}^{2} \leq \int_{\mathbb{S}^{n-1}} \sum_{a, b \in \mathbb{Z}}\left|\left\langle f, \Psi_{a, b, u}\right\rangle\right|^{2} d u \leq 2\|f\|_{2}^{2} .
$$

Especially for dimension two where the sphere is just the unit circle, it is easy to parametrize the sphere integral using angles $[0,2 \pi)$. The ridge function then corresponds with a rotation of the wavelet which has been extended constantly in the direction perpendicular to the angle.

We consider now some concrete examples. However, in some sense these examples act as a blueprint for the other types of frames.

Example 4.4. (Shannon wavelet) We consider the so-called Shannon wavelet,

$$
\psi(x):=\operatorname{sinc}(x)-\operatorname{sinc}(2 x)
$$

where

$$
\operatorname{sinc}(x):=\frac{\sin (\pi x)}{\pi x},
$$

see also $[28,7.2 .2]$. Clearly $\psi \in \mathcal{C}^{\infty}(\mathbb{R} ; \mathbb{C})$ because it is composed of two analytic functions, $\psi \in L^{2}$, but $\psi \notin L^{1}$. The spectrum $\mathcal{F}(\psi)(\omega)=-\chi_{[0.5,1]}(|\omega|)$ is compact. Thus $\psi \in H^{\alpha}(\mathbb{R} ; \mathbb{H})$ for any $\alpha \geq 0$. Therefore all requirements of Theorem 4.3 are fulfilled for lifting to any dimension. $\mathcal{D}^{\beta} \psi$ is bounded and continuous, and real and even for every choice of $\beta$ as $\psi$ is even and real.

A plot of $\psi$ and some of its pseudo derivatives is shown in Fig. 1. Its lifting to dimension two is shown in Fig. 2. 


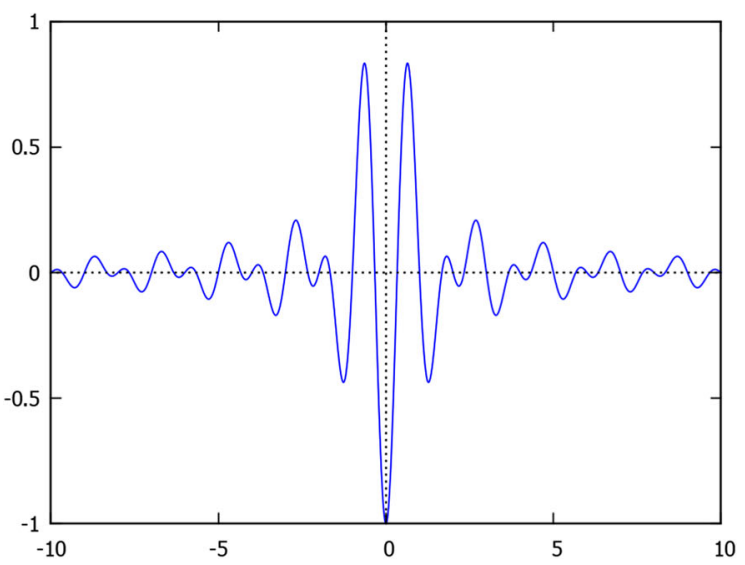

(a) The Shannon wavelet $\psi$

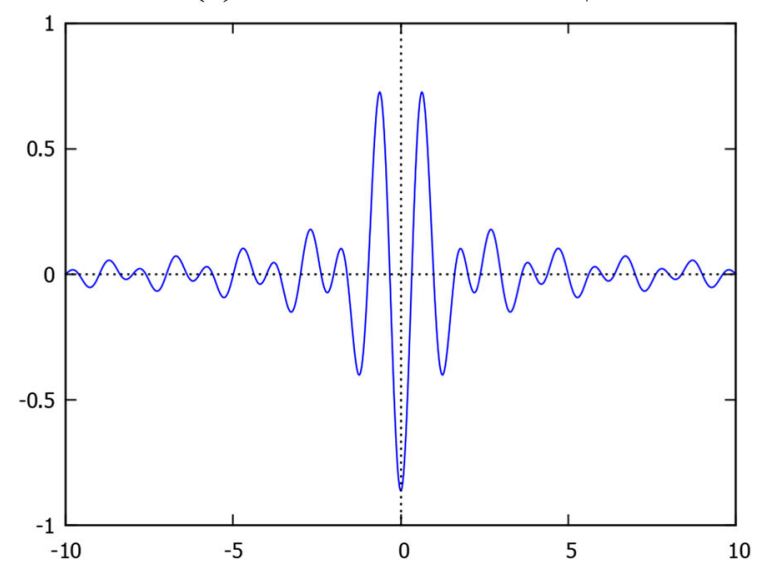

(b) $\mathcal{D}^{\frac{1}{2}} \psi$

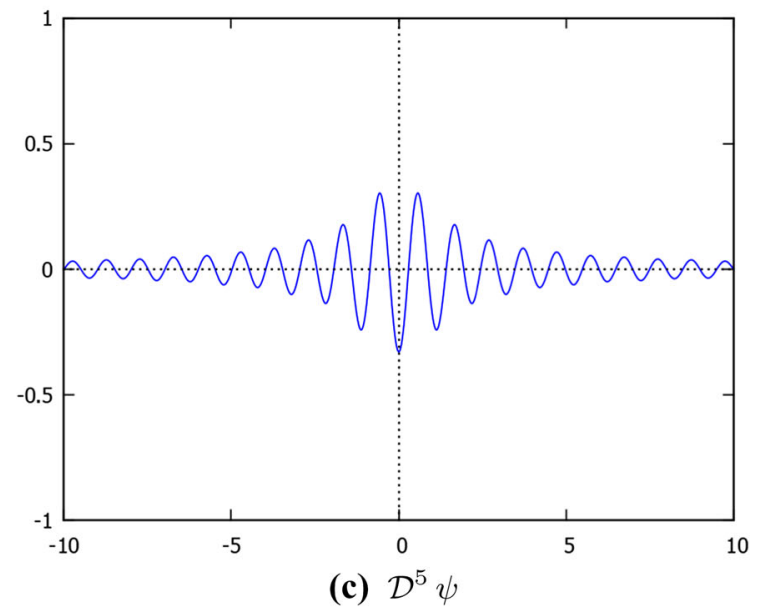

Figure 1. a The Shannon wavelet and $\mathbf{b}, \mathbf{c}$ some of its fractional derivatives. The derivatives remain real and even 


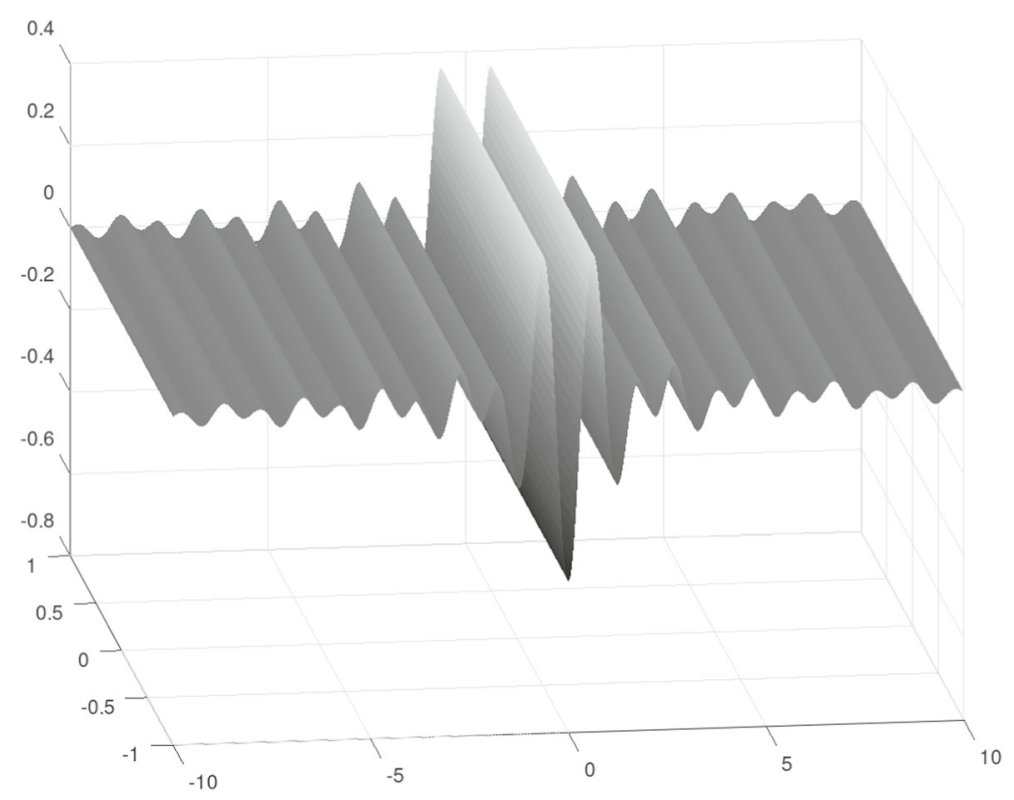

FiguRE 2. Lifted Shannon wavelet to dimension 2

\subsection{Shearlets}

Shearlets are a natural extension of wavelets to capture anisotropic features like edges and surface discontinuities in images. They are constructed by a parabolic scaling, a shearing and a translation. Unlike wavelets they do not constitute an orthonormal basis however they still form a frame. For further reading on shearlets we refer to [18] and [27]. Only recently shearlets have been extended to a quaternion setting in [31].

It will turn out again that it suffices to check the requirements of the lifting theorem only for the generating shearlet.

The shearlet structure is compatible with the pseudo differential operator for some special ridge constructions but not necessarily for all.

The following short introduction to quaternion-valued shearlets is based on [31] and uses the same notation - except if we have already introduced a different one. We consider two classes of matrices: For some $s \in \mathbb{R}$ the matrix

$$
S_{s}:=\left(\begin{array}{ll}
1 & s \\
0 & 1
\end{array}\right)
$$

is called shear matrix. And for some $a>0$ the matrix

$$
A_{a}:=\left(\begin{array}{cc}
a & 0 \\
0 & \sqrt{a}
\end{array}\right)
$$

is called parabolic scaling matrix. They act on functions $\psi: \mathbb{R}^{2} \rightarrow \mathbb{H}$ in the following way:

The shear operator for a shear matrix $S_{s}$ is defined as

$$
D_{S_{s}} \psi(x):=\psi\left(S_{s}^{-1} x\right)=\psi\left(S_{-s} x\right),
$$


and the parabolic scaling operator for a parabolic scaling matrix is given by

$$
D_{A_{a}} \psi(x):=\left|A_{a}\right|^{-\frac{1}{2}} \psi\left(A_{a}^{-1} x\right)=\left|A_{a^{-1}}\right|^{\frac{1}{2}} \psi\left(A_{a^{-1}} x\right) .
$$

Both operators are automorphisms of $L^{1}\left(\mathbb{R}^{2} ; \mathbb{H}\right)$ and of $L^{2}\left(\mathbb{R}^{2} ; \mathbb{H}\right)$.

Definition 4.5. (Admissibility condition) A function $\psi \in L^{2}\left(\mathbb{R}^{2} ; \mathbb{H}\right)$ is called shearlet if it satisfies the admissability condition

$$
C_{\psi}:=\int_{\mathbb{R}} \int_{\mathbb{R}}\left|\mathcal{F}_{S, i, j} \psi\left(\omega_{1}, \omega_{2}\right)\right|^{2} \frac{d \omega_{1} d \omega_{2}}{\omega_{1}^{2}}<\infty .
$$

For consistency with the [31] Definition 4.5 uses the sandwich version of the quaternionic Fourier transform. This choice does not influence the presented results as it does not matter how the frame was constructed.

For a shearlet $\psi \in L^{2}\left(\mathbb{R}^{2} ; \mathbb{H}\right)$, the quaternion shearlet system is defined as the family of functions

$$
\psi_{a, s, t}(x):=T_{t} D_{S_{s}} D_{A_{a}} \psi(x)=\left|A_{a}\right|^{-\frac{1}{2}} \psi\left(A_{a}^{-1} S_{s}^{-1}(x-t)\right),
$$

for $a \in \mathbb{R}^{+}, s \in \mathbb{R}, t \in \mathbb{R}^{2}$.

\section{Definition 4.6. (Shearlet transform)}

Let $\psi$ be a shearlet. For $f \in L^{2}\left(\mathbb{R}^{2} ; \mathbb{H}\right)$, the quaternion shearlet transform is defined as

$$
S_{\psi} f(a, s, t):=\left\langle f, \psi_{a, s, t}\right\rangle
$$

for almost all $a \in \mathbb{R}^{+}, s \in \mathbb{R}, t \in \mathbb{R}^{2}$.

Theorem 4.7. Let $\psi \in L^{2}\left(\mathbb{R}^{2} ; \mathbb{H}\right)$ be a shearlet that satisfies the admissibility condition. Then for every $f \in L^{2}\left(\mathbb{R}^{2} ; \mathbb{H}\right)$,

$$
\int_{\mathbb{R}^{2}} \int_{\mathbb{R}} \int_{\mathbb{R}^{+}}\left|\left\langle f, \psi_{a, s, t}\right\rangle\right|^{2} \frac{d a d s d t}{a^{3}}=C_{\psi}\|f\|_{2}^{2} .
$$

Proof. See [31].

We show that the parabolic scaling and the shear operator have an exploitable relation with the Fourier transform.

Lemma 4.8. (Fourier relation) Let $f \in L^{1}\left(\mathbb{R}^{2} ; \mathbb{H}\right)$ and $s \in \mathbb{R}$ and $a>0$ then

(i) $\mathcal{F}\left(D_{S_{s}} f\right)=D_{S_{-s}^{T}} \mathcal{F} f$.

(ii) $\mathcal{F}\left(D_{A_{a}} f\right)=D_{A_{a-1}} \mathcal{F} f$.

Proof. Direct calculation.

Now these relations can be used to show that the parabolic scaling and the shear operator are automorphisms on $H\left(\mathbb{R}^{2} ; \mathbb{H}\right)$ and $\mathcal{S}\left(\mathbb{R}^{2} ; \mathbb{H}\right)$.

Lemma 4.9. (i) $\psi \in H^{\alpha}\left(\mathbb{R}^{2} ; \mathbb{H}\right) \Leftrightarrow D_{S_{s}} \psi \in H^{\alpha}\left(\mathbb{R}^{2} ; \mathbb{H}\right)$

$$
\text { (ii) } \psi \in H^{\alpha}\left(\mathbb{R}^{2} ; \mathbb{H}\right) \Leftrightarrow D_{A_{a}} \psi \in H^{\alpha}\left(\mathbb{R}^{2} ; \mathbb{H}\right)
$$


Proof. (i) “ $\Rightarrow$ " Using Theorem 2.4, Lemma 4.8 and integration by substitution yields

$$
\begin{aligned}
& \int_{\mathbb{R}^{2}}|\omega|^{2 \alpha}\left|\mathcal{F} D_{S_{s}} f(\omega)\right|^{2} d \omega=\int_{\mathbb{R}^{2}}|\omega|^{2 \alpha}\left|D_{S_{-s}^{T}} \mathcal{F} f(\omega)\right|^{2} d \omega \\
& \quad=\int_{\mathbb{R}^{2}}|\omega|^{2 \alpha}\left|\mathcal{F} f\left(S_{-s}^{T} \omega\right)\right|^{2} d \omega=\int_{\mathbb{R}^{2}}\left|S_{s}^{T} \omega\right|^{2 \alpha}|\mathcal{F} f(\omega)|^{2} d \omega \\
& \quad \stackrel{(1)}{\leq}(1+|s|)^{2 \alpha} \int_{\mathbb{R}^{2}}|\omega|^{2 \alpha}|\mathcal{F} f(\omega)|^{2} d \omega<\infty
\end{aligned}
$$

where (1) follows from

$$
\begin{aligned}
\left|S_{s}^{T} \omega\right| & =\left|\left(\begin{array}{c}
\omega_{1} \\
s \omega_{1}+\omega_{2}
\end{array}\right)\right| \leq\left|\left(\begin{array}{c}
\omega_{1} \\
\omega_{2}
\end{array}\right)\right|+\left|\left(\begin{array}{c}
0 \\
s \omega_{2}
\end{array}\right)\right| \\
& \leq(1+|s|)\left|\left(\begin{array}{c}
\omega_{1} \\
\omega_{2}
\end{array}\right)\right|=(1+|s|)|\omega| .
\end{aligned}
$$

" $\Leftarrow "$ Again by integration by substitution

$$
\begin{aligned}
\int_{\mathbb{R}^{2}}|\omega|^{2 \alpha}|\mathcal{F} f(\omega)|^{2} d \omega & =\int_{\mathbb{R}^{2}}\left|S_{-s}^{T} \omega\right|^{2 \alpha}\left|\mathcal{F} f\left(S_{-s}^{T} \omega\right)\right|^{2} d \omega \\
& \leq(1+|s|)^{2 \alpha} \int_{\mathbb{R}^{2}}|\omega|^{2 \alpha}\left|\mathcal{F} f\left(S_{-s}^{T} \omega\right)\right|^{2} d \omega \\
& =(1+|s|)^{2 \alpha} \int_{\mathbb{R}^{2}}|\omega|^{2 \alpha}\left|\mathcal{F} D_{S_{s}} f(\omega)\right|^{2} d \omega<\infty .
\end{aligned}
$$

(ii) This proof is similar to dilation in the wavelet case and to the previous one. We just pull out the factor $\max (a, \sqrt{a})$ after performing a integration.

Hence it suffices to show that the generating functions fulfill the requirements for the lifting procedure.

Corollary 4.10. Let $\psi \in L^{2}\left(\mathbb{R}^{2} ; \mathbb{H}\right)$ be a shearlet.

$$
\psi \in H^{\alpha}\left(\mathbb{R}^{2} ; \mathbb{H}\right) \Leftrightarrow\left\{\psi_{a, s, t}\right\}_{a \in \mathbb{R}^{+}, s \in \mathbb{R}, t \in \mathbb{R}^{2}} \subset H^{\alpha}\left(\mathbb{R}^{2} ; \mathbb{H}\right) .
$$

Unfortunately the pseudo differential operator only commutes with the parabolic scaling but not with the shear because

$$
\mathcal{F}\left(D_{S_{s}} f\right)\left(\omega_{1}, \omega_{2}\right)=D_{S_{-s}} \mathcal{F} f\left(\omega_{1}, \omega_{2}\right)=\mathcal{F} f\left(\omega_{1}+s \omega_{2}, \omega_{2}\right) .
$$

The application of $\mathcal{D}$ in Fourier domain yields the factor $|\omega|^{\beta}$. To make the Fourier transform independent of the shear a substitution has to be made. Unfortunately this yields a undesired difference in $|\omega|^{\beta}$. To overcome this problem we restrict the flexibility of the approach in favor of compatibility and set $\beta_{2}=0$. I.e we only allow lifting in the second argument.

Lemma 4.11. Let $\beta=\left(\beta_{1}, 0\right), \psi \in H^{\alpha}\left(\mathbb{R}^{2} ; \mathbb{H}\right)$ be a suitable shearlet. Then

$$
\mathcal{D}^{\beta}\left(D_{S_{s}} \psi\right)=D_{S_{s}} \mathcal{D}^{\beta} \psi \text {. }
$$


Proof.

$$
\mathcal{D}^{\beta}\left(D_{S_{s}} \psi\right)=\mathcal{F}^{-1}\left(D_{S_{-s}^{T}} \mathcal{F} \psi|\cdot|^{\beta}\right)=\mathcal{F}^{-1}\left(D_{S_{-s}^{T}}\left(\mathcal{F} \psi|\cdot|^{\beta}\right)\right)=D_{S_{s}} \mathcal{D}^{\beta} \psi
$$

where we exploited $\beta_{2}=0$, because now we substitute $\left|\omega_{2}\right|^{\beta_{1}}$ by $\left|s \omega_{1}+\omega_{2}\right|^{\beta_{2}}$.

For the parabolic scaling one gets:

Lemma 4.12. Let $\psi \in H^{\alpha}\left(\mathbb{R}^{2} ; \mathbb{H}\right)$ be a suitable shearlet. Then

$$
\mathcal{D}^{\beta}\left(D_{A_{a}} \psi\right)=a^{-\beta_{1}-\frac{1}{2} \beta_{2}} D_{A_{a}} \mathcal{D}^{\beta} \psi .
$$

Proof.

$$
\begin{array}{r}
\mathcal{D}^{\beta}\left(D_{A_{a}} \psi\right)=\mathcal{F}^{-1}\left(\mathcal{F}\left(D_{A_{a}} \psi\right)|\cdot|^{\beta}\right)=\mathcal{F}^{-1}\left(D_{A_{a}-1} \mathcal{F}(\psi)|\cdot|^{\beta}\right) \\
\quad=\mathcal{F}^{-1}\left(D_{A_{a-1}}\left(\left.\mathcal{F}(\psi) a^{-\beta_{1}-\frac{1}{2} \beta_{2}}|\cdot|\right|^{\beta}\right)\right)=a^{-\beta_{1}-\frac{1}{2} \beta_{2}} D_{A_{a}} \mathcal{D}^{\beta} \psi
\end{array}
$$

In summary, the interpretation of the shearlet coefficients lifts for any $\beta=\left(\beta_{1}, 0\right)$. Clearly this limits the flexibility, however we consider compatibility of the structure with the lifting technique to be more valuable than the flexibility in the choice of $\beta$.

Corollary 4.13. Let $\psi \in H^{\alpha}\left(\mathbb{R}^{2} ; \mathbb{H}\right)$ such that $\alpha \geq \frac{n-1}{2}$. Then

$$
\mathcal{D}^{\frac{n-1}{2}, 0} \psi_{a, s, t}=a^{-\frac{n-1}{2}} T_{t} D_{S_{s}} D_{A_{a}} \mathcal{D}^{\frac{n-1}{2}, 0} \psi .
$$

In this case it also suffices to prove the requirements only for the generating function and not for the whole frame.

Corollary 4.14. For dimension $n \in \mathbb{N}$ and a shearlet $\psi \in H^{\alpha}\left(\mathbb{R}^{2} ; \mathbb{H}\right)$ such that $\alpha \geq \frac{n-1}{2}$ it holds

$$
\mathcal{D}^{\frac{n-1}{2}, 0} \psi \in L^{\infty}\left(\mathbb{R}^{2} ; \mathbb{H}\right) \Leftrightarrow \mathcal{D}^{\frac{n-1}{2}, 0} \psi_{a, s, t} \in L^{\infty}\left(\mathbb{R}^{2} ; \mathbb{H}\right)
$$

for any $a \in \mathbb{R}^{+}, s \in \mathbb{R}, t \in \mathbb{R}^{2}$.

The much easier $L^{1}\left(\mathbb{R}^{2} ; \mathbb{H}\right)$ criterion is also present:

Corollary 4.15. Let $\psi \in H^{\alpha}\left(\mathbb{R}^{2} ; \mathbb{H}\right)$ and $0 \leq \beta_{1} \leq \alpha$ then

$$
\mathcal{F} \psi\left(\omega_{1}, \omega_{2}\right)\left|\omega_{1}\right|^{\beta_{1}} \in L^{1}\left(\mathbb{R}^{2} ; \mathbb{H}\right) \Leftrightarrow \mathcal{F} \psi_{a, s, t}\left(\omega_{1}, \omega_{2}\right)\left|\omega_{1}\right|^{\beta} \in L^{1}\left(\mathbb{R}^{2} ; \mathbb{H}\right)
$$

for any $a \in \mathbb{R}^{+}, s \in \mathbb{R}, t \in \mathbb{R}^{2}$. In this case $\mathcal{D}^{\beta} \psi_{a, s, t} \in C_{0}\left(\mathbb{R}^{2} ; \mathbb{H}\right)$ for all $a \in \mathbb{R}^{+}, s \in \mathbb{R}, t \in \mathbb{R}^{2}$.

Similar to the wavelet case the lifting remains completely real if the generating function is real-valued.

Corollary 4.16. Let $\psi \in H^{\alpha}\left(\mathbb{R}^{2} ; \mathbb{H}\right)$ a shearlet, $n \in \mathbb{N}$ the dimension to lift to and $\alpha \geq \frac{n-1}{2}$. Then it holds that

$$
\psi\left(\mathbb{R}^{2}\right) \subset \mathbb{R} \Rightarrow \mathcal{D}^{\frac{n-1}{2}, 0}\left(\psi_{a, s, t}\right)\left(\mathbb{R}^{2}\right) \subset \mathbb{R}
$$


To illustrate the results above we give two examples to construct a quaternion shearlet due to the lack of concrete examples in common literature on quaternion shearlets. The first will be a classic shearlet-like example and the second one a upcycling approach which generalizes real valued shearlets to quaternion valued ones.

Example 4.17. For a pure unit quaternion $\mu$ and the unit ball $B$ on $\mathbb{R}^{2}$ let

$$
\mathcal{F}_{S, \mu_{1}, \mu_{2}} \psi\left(\omega_{1}, \omega_{2}\right):=\omega_{1} e^{\mu \omega_{1}} e^{\frac{-1}{1-\|\omega\|^{2}}} \cdot \chi_{B}(\omega),
$$

where $\chi_{B}$ is the characteristic function of $B$. Then the function $\mathcal{F}_{S, \mu_{1}, \mu_{2}} \psi$ is smooth, bounded and has compact support. Hence

$$
\int_{\mathbb{R}} \int_{\mathbb{R}}\left|\mathcal{F}_{S, \mu_{1}, \mu_{2}} \psi\left(\omega_{1}, \omega_{2}\right)\right|^{2} \frac{d \omega_{1} d \omega_{2}}{\omega_{1}^{2}}=\int_{B}\left|e^{\frac{-1}{1-\|\omega\|^{2}}}\right|^{2} d \omega<\infty .
$$

As the support of the $\mathcal{F}_{S, \mu_{1}, \mu_{2}} \psi$ is compact, $\psi \in H^{\infty}\left(\mathbb{R}^{2} ; \mathbb{H}\right)$ and can therefore be used for lifting to any dimension with any distribution of the lifted dimensions to the two variables.

Example 4.18. Let $\psi_{i} \in H^{\alpha}\left(\mathbb{R}^{2} ; \mathbb{R}\right)$ for $i=1, \ldots, 4$ be real shearlets then $\psi:=\psi_{1}+i \psi_{2}+j \psi_{3}+k \psi_{4}$ is a quaternionic shearlet.

\section{Conclusion}

In our paper we gave a method to raise one-dimensional frames to higher dimensions in quaternionic-valued Hilbert spaces. The idea was the ridge construction together with the use of fractional differential operators as it has been introduced for Gabor frames. The advantage of the technique is that the frame bounds from the one-dimensional case can be carried over to the multidimensional case by simple multiplication with a constant factor depending on the dimension only. The technique can also be applied to wavelet frames. In our article, we extended the technique to the non-commutative quaternionic setting and considered multi-ridges. They allow to group the dimensions of a vector space into subspaces and to treat each subspace differently with regard to the frame lifting. This can be helpful in cases when not all available dimensions are of the same category of interpretation, e.g. time-, space- and spectral dimensions in image processing. We based our analysis on the widely used quaternionic Fourier transform generated from quaternionifying the complex exponential Fourier kernel. We defined the corresponding Sobolev/Bessel potential spaces to be able to apply the fractional derivatives in this setting and proved similarities and differences of these spaces with regard to the variety of the Fourier transform family. The method can be applied to a plethora a frame families, such as Gabor frames, wavelets, shearlets, and others.

Funding Open Access funding enabled and organized by Projekt DEAL. 
Open Access. This article is licensed under a Creative Commons Attribution 4.0 International License, which permits use, sharing, adaptation, distribution and reproduction in any medium or format, as long as you give appropriate credit to the original author(s) and the source, provide a link to the Creative Commons licence, and indicate if changes were made. The images or other third party material in this article are included in the article's Creative Commons licence, unless indicated otherwise in a credit line to the material. If material is not included in the article's Creative Commons licence and your intended use is not permitted by statutory regulation or exceeds the permitted use, you will need to obtain permission directly from the copyright holder. To view a copy of this licence, visit http:// creativecommons.org/licenses/by/4.0/.

Publisher's Note Springer Nature remains neutral with regard to jurisdictional claims in published maps and institutional affiliations.

\section{References}

[1] Ali, S.T., Antoine, J.-P., Jean-Pierre, G.: Continuous frames in Hilbert space. Ann. Phys. 222(1), 1-37 (1993)

[2] Bahri, M.: Construction of quaternion-valued wavelets. Matematika 26(1), 107$114(2010)$

[3] Bahri, M., Hitzer, E., Hayashi, A., Ashino, R.: An uncertainty principle for quaternion Fourier transform. Comput. Math. Appl. 56(9), 2398-2410 (2008)

[4] Bayro-Corrochano, E.: The theory and use of the quaternion wavelet transform. J. Math. Imaging Vis. 24, 19-35 (2006)

[5] Bernstein, S. (ed.): Topics in Clifford Analysis. Birkhäuser, Basel (2019)

[6] Bülow, T.: Hypercomplex Spectral Signal Representations for the Processing and Analysis of Images. Institut für Informatik und Praktische Mathematik, Christian-Albrechts-Universität Kiel, Bericht (1999)

[7] Carré, P., Denis, P.: Quaternionic wavelet transform for colour images. In: Proceedings of SPIE-The International Society for Optical Engineering, October (2006)

[8] Cheng, D., Kou, K.I.: Plancherel theorem and quaternion Fourier transform for square integrable functions. Complex Var. Elliptic Equ. 64, 223-42 (2018)

[9] Christensen, O.: An Introduction to Frames and Riesz Bases, 1st edn. Birkhäuser Mathematics, Basel (2003)

[10] Christensen, O., Forster, B., Massopust, P.: Directional time-frequency analysis via continuous frames. Bull. Aust. Math. Soc. 92, 268-281 (2015)

[11] Conway, J., Smith, D.: On Quaternions and Octonions. Taylor \& Francis Inc, Milton Park (2001)

[12] De Bie, H., De Schepper, N., Sommen, F.: The class of Clifford-Fourier transforms. J. Fourier Anal. Appl. 17, 1198-1231 (2011)

[13] Ell, T.A., Sangwine, S.J.: Hypercomplex Fourier transforms of color images. IEEE Trans. Image Process. 16(1), 22-35 (2007)

[14] Ell, T., Sangwine, S.: Hypercomplex Fourier transforms of color images. IEEE Trans. Image Process. 16(1), 22-35 (2007)

[15] Ell, T.A., Bihan, N.L., Sangwine, S.J.: Quaternion Fourier Transforms for Signal and Image Processing. Wiley-ISTE, London (2014) 
[16] Fletcher, P., Sangwine, S.J.: The development of the quaternion wavelet transform. Signal Process. 136, 2-15 (2017)

[17] Grafakos, L., Sansing, C.: Gabor frames and directional time-frequency analysis. Appl. Comput. Harm. Anal. 25(1), 47-67 (2008)

[18] Guo, K., Labate, D.: Optimally sparse multidimensional representation using shearlets. SIAM J. Math. Anal. 39(1), 298-318 (2007)

[19] Haroske, D.D., Triebel, H.: Distributions, Sobolev Spaces. EMS Textbooks in Mathematics, Elliptic Equations (2007)

[20] He, J.-X.: Wavelet analysis of quaternion-valued time-series. Int. J. Wavel. Multiresolut. Inf. Process. 3(2), 233-246 (2005)

[21] Held, S., Storath, M., Massopust, P., Forster, B.: Steerable wavelet frames based on the Riesz transform. IEEE Trans. Image Process. 19(3), 653-667 (2010)

[22] Hitzer, E., Sangwine, S.J. (eds.): Quaternion and Clifford Fourier Transforms and Wavelets. Birkhäuser, Basel (2013)

[23] Hogan, J.A., Joel Morris, A.: Quaternionic wavelets. Numer. Funct. Anal. Optim. 33(7-g), 1031-1062 (2012)

[24] Kaiser, G.: A Friendly Guide to Wavelets. Birkhäuser Boston, Boston (2011)

[25] Katznelson, Y.: An Introduction to Harmonic Analysis. Cambridge University Press, Cambridge (2004). (3rd edition)

[26] Kon, M.A., Raphael, L.A.: A characterization of wavelet convergence in Sobolev spaces. Appl. Anal. 78, 271-324 (2001)

[27] Kutyniok, G., Wang-Q, L.: Compactly supported shearlets are optimally sparse. J. Approx. Theory 163(11), 1564-1589 (2011)

[28] Mallat, S.: A Wavelet Tour of Signal Processing: The Sparse Way. Elsevier Academic Press, Amsterdam (2009)

[29] Natterer, F.: The Mathematics of Computerized Tomography. Teubner B. G., Stuttgart (1986)

[30] Sangwine, S.J., Horne, R.E.N. (eds.): The Colour Image Processing Handbook. Springer, Berlin (1998)

[31] Shah, F.A., Tantary, A.Y.: On quaternion shearlet transforms. arXiv preprint arXiv:1803.04248 (2018)

[32] Triebel, H.: Theory of Function Spaces II. Birkhäuser, Basel (1992)

[33] Triebel, H.: Theory of Function Spaces. Springer Science, Berlin (2010)

[34] Unser, M., Van De Ville, D.: Wavelet steerability and the higher-order Riesz transform. Trans. Image Process. 19(3), 636-652 (2010)

[35] Werner, D.: Funktional Analysis, 7th edn. Springer, Berlin (2011)

Florian Heinrich

Fakultät für Informatik und Mathematik

Universität Passau

Innstraße 43

94032 Passau

Germany

e-mail: florian.heinrich@uni-passau.de 


\section{Brigitte Forster}

Fakultät für Informatik und Mathematik

Universität Passau

Innstr. 33

94032 Passau

Germany

e-mail: brigitte.forster@uni-passau.de

Received: January 28, 2020.

Accepted: October 17, 2020. 